\title{
SIGNATURE CATALAN COMBINATORICS
}

\author{
CESAR CEBALLOS AND RAFAEL S. GONZÁLEZ D'LEÓN
}

\begin{abstract}
The Catalan numbers constitute one of the most important sequences in combinatorics. Catalan objects have been generalized in various directions, including the classical Fuss-Catalan objects and the rational Catalan generalization of Armstrong-RhoadesWilliams. We propose a wider generalization of these families indexed by a composition $s$ which is motivated by the combinatorics of planar rooted trees; when $s=(2, \ldots, 2)$ and $s=(k+1, \ldots, k+1)$ we recover the classical Catalan and Fuss-Catalan combinatorics, respectively. Furthermore, to each pair $(a, b)$ of relatively prime numbers we can associate a signature that recovers the combinatorics of rational Catalan objects. We present explicit bijections between the resulting $s$-Catalan objects, and a fundamental recurrence that generalizes the fundamental recurrence of the classical Catalan numbers. Our framework allows us to define signature generalizations of parking functions which coincide with the generalized parking functions studied by Pitman-Stanley and Yan, as well as generalizations of permutations which coincide with the notion of Stirling multipermutations introduced by Gessel-Stanley. Some of our constructions differ from the ones of Armstrong-Rhoades-Williams, however as a byproduct of our extension, we obtain the additional notions of rational permutations and rational trees.
\end{abstract}

\section{Contents}

1. Introduction 2

2. Preliminaries 4

2.1. Compositions and weak compositions 4

2.2. Catalan objects 5

2.3. $s$-Catalan objects 6

3. The $s$-Catalan zoo and bijections $\quad 7$

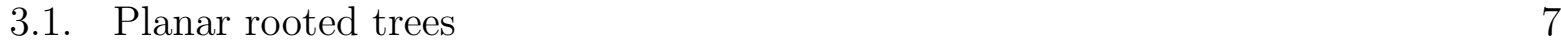

3.2. Dyck paths 10

3.3. 312-avoiding Stirling permutations $\quad 15$

3.4. Noncrossing partitions 19

3.5. Noncrossing matchings 23

4. The fundamental recurrence 26

4.1. Catalan decompositions 26

C. Ceballos was supported by the Austrian Science Foundation FWF, grant F 5008-N15, in the framework of the Special Research Program Algorithmic and Enumerative Combinatorics"; he was also partially supported by York University and a Banting Postdoctoral Fellowship of the Government of Canada. R. S. González D'León was supported during this project by University of Kentucky, York University and Universidad Sergio Arboleda and he is grateful for their support. 
4.2. Catalan decomposition for Dyck paths 27

4.3. Angulations of a polygon 28

4.4. Parenthesizations 28

5. Enumeration 30

5.1. $s$-Catalan numbers 30

5.2. s-Narayana numbers 30

6. Signature generalizations of permutations and parking functions 31

6.1. s-generalized permutations 31

6.2. $s$-generalized parking functions $\quad 32$

7. Relation to the rational constructions of Armstrong, Rhoades and Williams 34

8. Algebraic structures on signature objects 35

Appendix A. Bijections with $s$-Dyck paths 38

A.1. $s$-Dyck paths and 312-avoiding Stirling $(s-\mathbf{1})$-permutations 38

A.2. $s$-Dyck paths and noncrossing $(s-\mathbf{1})$-partitions 38

A.3. $s$-Dyck paths and complete noncrossing $s$-matchings $\quad 40$

$\begin{array}{ll}\text { Acknowledgements } & 40\end{array}$

$\begin{array}{ll}\text { References } & 40\end{array}$

\section{INTRODUCTION}

A permutation $\sigma$ of the set $[n]:=\{1,2, \ldots, n\}$ is a bijection $\sigma:[n] \rightarrow[n]$. A permutation can be represented in the one-line notation $\sigma_{1} \sigma_{2} \cdots \sigma_{n}$ where $\sigma_{i}:=\sigma(i)$. We denote the set of permutations of $[n]$ by $\mathfrak{S}_{n}$. This set has the structure of a group under composition of permutations and it is commonly known as the symmetric group. It is an introductory exercise in enumerative combinatorics to show that the cardinality of $\mathfrak{S}_{n}$ is given by the factorial numbers $n !:=1 \cdot 2 \cdots(n-1) \cdot n$. There are other families of objects that are in bijection with permutations. For example, there is a bijection between $\mathfrak{S}_{n}$ and the set $\mathcal{I T}_{n}$ of binary trees drawn in the plane with a distinguished vertex or root and with labels in the internal nodes that are increasing when walking away from the root. The trees in $\mathcal{I}_{n}$ are also known as increasing rooted planar binary trees (see [34, Chapter 1] for the bijection and Figure 1 for an example of the bijection when $n=3$ ). We will be denoting by $\mathcal{S}_{n}$ a generic family of objects that is in bijection with $\mathfrak{S}_{n}$, that is, $\left|\mathcal{S}_{n}\right|=n$ !.

We say that a permutation $\sigma$ is 312-avoiding if there are no indices $i<j<k$ such that $\sigma_{j}<\sigma_{k}<\sigma_{i}$. We denote by $\mathfrak{S}_{n}(312)$ the set of 312 -avoiding permutations in $\mathfrak{S}_{n}$. It is known that the set $\mathfrak{S}_{n}(312)$ is in bijection with the set $\mathcal{D} \mathcal{P}_{n}$ of lattice paths in $\mathbb{N} \times \mathbb{N}$ from $(0,0)$ to $(n, n)$ taking only north steps $(0,1)$ and east steps $(1,0)$, and such that at any given point the number of north steps taken is greater than or equal to the number of east steps taken (see Figure 1 for an example of the bijection when $n=3$ ). These lattice paths are also known as Dyck paths. Any family of objects in bijection with either $\mathfrak{S}_{n}(312)$ or $\mathcal{D} \mathcal{P}_{n}$ is known as a Catalan family named after the Belgian mathematician Eugène Charles Catalan who studied them. Catalan objects are probably the most intriguing objects in combinatorics since they appear in connection with numerous fields of mathematics in 
many different forms. Many mathematicians have studied enumerative, geometric and algebraic occurrences of the Catalan families. In particular, Richard Stanley has collected and curated a selection of these families during years of study [35]. Let us denote by $\mathcal{C}_{n}$ a generic family of Catalan objects. It is known that $\left|\mathcal{C}_{n}\right|=\frac{1}{n+1}\left(\begin{array}{c}2 n \\ n\end{array}\right)=\frac{1}{2 n+1}\left(\begin{array}{c}2 n+1 \\ n\end{array}\right)=\frac{(2 n) !}{(n+1) ! n !}$ what is known as the $n$-th Catalan number.

From objects in $\mathcal{D P}{ }_{n}$ we can create a different family $\widehat{\mathcal{D P}}_{n}$ of objects obtained by decorating the north steps of a Dyck path with a permutation of $[n]$ in such a way that the consecutive sequences of north steps (also known as runs) have increasing values from bottom to top (see Figure 1). The objects obtained in this way are known as decorated Dyck paths and happen to be in bijection with another famous family of combinatorial objects known as parking functions. The set $\mathcal{P} \mathcal{F}_{n}$ of parking functions of $n$ consists of sequences of $n$ nonnegative integers $\left(p_{1}, p_{2}, \ldots, p_{n}\right)$ with the property that after being rearranged in weakly increasing order $p_{j_{1}} \leq p_{j_{2}} \leq \cdots \leq p_{j_{n}}$ they satisfy $p_{j_{i}}<i$ for all $i \in[n]$. Figure 1 shows an example of the bijection between decorated Dyck paths and parking functions for $n=3$. Parking functions were studied by Konheim and Weiss [14] using a different description that explains their name. There are other popular families of objects in bijection with $\mathcal{P} \mathcal{F}_{n}$, for example, the set of (nonplanar nonrooted) trees on the vertex set $\{0\} \cup[n]$ is in bijection with $\mathcal{P F}_{n}$ (see $[12,15,28]$ ). Let us denote any generic family of objects in bijection with $\mathcal{P} \mathcal{F}_{n}$ by $\mathcal{P}_{n}$. It is also known that $\left|\mathcal{P}_{n}\right|=(n+1)^{n-1}$, see for example [33].

The families counted by the sequences $n !, \frac{1}{n+1}\left(\begin{array}{c}2 n \\ n\end{array}\right)$ and $(n+1)^{n-1}$ for $n \geq 0$ have intrigued mathematicians for centuries and in a nutshell intuitively we can describe their relation by the following idea.

Idea 1. A family $\mathcal{C}_{n}$ is obtained as a sub-family of $\mathcal{S}_{n}$ satisfying a suitable restriction. $A$ family $\mathcal{P}_{n}$ can be obtained by extending a family $\mathcal{C}_{n}$ with a decoration of its objects with the elements of $[n]$, also subject to a suitable restriction on the decoration.

Idea 1 is the underlying idea in the story of the classical combinatorial objects mentioned above. As mentioned in [3], there are two general directions in which we can generalize this story. The first considers that all the families of objects discussed, $\mathcal{S}_{n}, \mathcal{C}_{n}$ and $\mathcal{P}_{n}$, are related directly to the combinatorics of the symmetric group that happens to be the Weyl group of Coxeter type A. Hence, we can wonder if there are corresponding objects for other Coxeter types. Work in this direction has been done by some authors like Reiner [31], Athanasiadis [4], Fomin and Reading [7], Armstrong [1], Williams [37] and others, and it is currently an active area of research. The second direction to generalize these families of objects is to consider Catalan objects as a phenomenon that depends on two relatively prime numbers $a, b$ such that a family $\mathcal{C}_{a, b}$ parametrized by these numbers has cardinality $\frac{1}{a+b}\left(\begin{array}{c}a+b \\ b\end{array}\right)$, also known as the rational Catalan number. When $a=n$ and $b=n+1$ we recover the classical Catalan numbers, and when $a=n$ and $b=k n+1$ we obtain another classical generalization known as the Fuss-Catalan numbers. Even thought ingredients of the rational Catalan story have appeared previously in the literature, Armstrong, Rhodes and Williams started a more systematic study of the rational Catalan objects $\mathcal{C}_{a, b}$ in [3]. In this generalization of the classical story there are rational versions $\mathcal{P}_{a, b}$ of the parking 


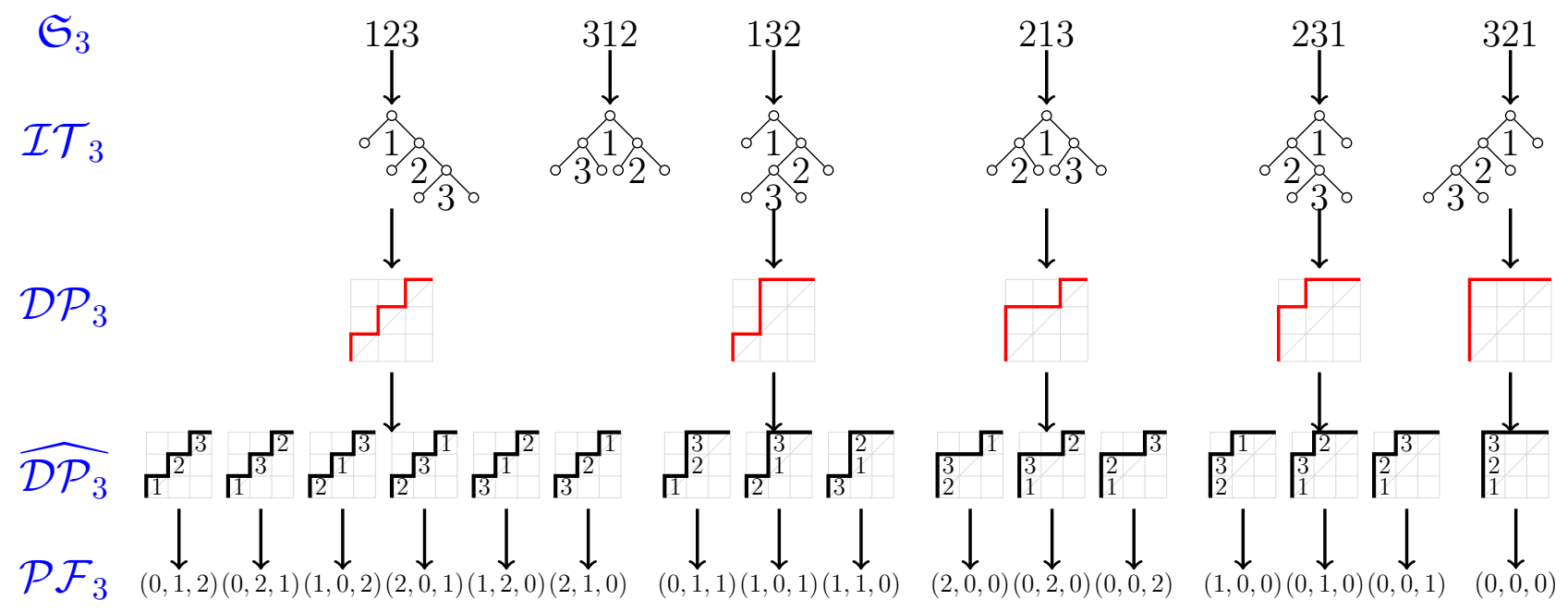

Figure 1. Maps between classical combinatorial objects in type A

objects in $\mathcal{P}_{n}$ (see for example [2]), but it is not clear what is the generalization for the permutation objects in $\mathcal{S}_{n}$ to a rational version $\mathcal{S}_{a, b}$.

In this paper we start a systematic study of a further generalization of the rational Catalan objects. Our generalization is indexed by a composition $s=\left(s_{1}, s_{2}, \ldots, s_{a}\right)$ that we call a signature. When $s=(2, \ldots, 2)$ and $s=(k+1, \ldots, k+1)$ we recover the classical Catalan and Fuss-Catalan objects respectively. The rational Catalan objects are obtained by the signature whose $i$-th entry is the number of boxes in the $i$-th row of an $b \times a$ grid that are crossed by the main diagonal of the grid.

The central idea for our generalization of Catalan objects relies on the combinatorics of planar rooted trees. For a given signature $s$ we associate a family $\mathcal{T}_{s}$ of planar rooted trees that we call $s$-trees. Based on their structural properties one can obtain generalizations of other classical Catalan objects such as Dyck paths, 312-avoiding permutations, noncrossing partitions, complete noncrossing matchings, triangulations of a polygon, and parenthesizations. We also propose generalizations of permutations and parking functions objects in this general set up. The generalized permutations are encoded by a family of increasingly labeled planar rooted trees. These labeled trees are in bijection with a generalization of the Stirling permutations studied by Gessel and Stanley back in the seventies [10] (see also $[27,26,25,11,13,17,24,6,32])$. The generalized parking functions are obtained as appropriate decorations of $s$-Catalan objects as it is classically done in the case of rational parking functions [2]. These also coincide with the ones originally studied by Pitman and Stanley [36] and Yan $[39,38]$ in a slightly more general form.

\section{Preliminaries}

2.1. Compositions and weak compositions. We denote by $\mathbb{P}$ the set of positive integers and by $\mathbb{N}$ the set of nonnegative integers. A weak composition is a finite sequence $\mu=$ 
$(\mu(1), \mu(2), \ldots, \mu(\ell))$ of numbers $\mu(i) \in \mathbb{N}$. For a weak composition $\mu$ we define its sum $|\mu|:=\sum_{i} \mu(i)$ and its length $\ell(\mu):=\ell$. For example for $\mu=(2,0,3,4,0,1)$ we have that $\ell(\mu)=6$ and $|\mu|=10$. If $|\mu|=n$ for some $n \in \mathbb{N}$, we say that $\mu$ is a weak composition of $n$. We denote by $\mathcal{W C o m p}$ the set of weak compositions and $\mathcal{W C}$ omp $p_{n}$ the set of weak compositions of $n$. A composition is a weak composition $\mu$ such that $\mu(i) \neq 0$ for all $i \in[\ell]$, in other words, a composition is a finite sequence of entries in $\mathbb{P}$. We denote by $\mathcal{C}$ omp the set of compositions and $\mathcal{C}_{\text {omp }}$ the set of compositions of $n$. An (integer) partition $\lambda$ of $n$ (denoted $\lambda \vdash n$ ) is a composition of $n$ whose entries are nonincreasing, i.e., $\lambda=(\lambda(1) \geq \lambda(2) \geq \cdots)$. We denote by $\mathcal{P}$ ar the set of partitions and $\mathcal{P}_{a} r_{n}$ the set of partitions of $n$.

We can define several partial orders on the set of (weak) compositions. We introduce the two orderings that we will be using in this article. For $\mu, \nu \in \mathcal{W C}$ omp we say that $\mu$ is a refinement of $\nu$ if $\nu$ can be obtained from $\mu$ by adding adjacent parts. For example, $(3,2,1,1,5,2,2)$ is a refinement of $(6,1,7,2)$ since $(6,1,7,2)=(3+2+1,1,5+2,2)$. Refinement defines a partial order in $\mathcal{W C O m p}$ (also in $\mathcal{C}$ omp) and we say that $\mu \leq \nu$ if $\mu$ is a refinement of $\nu$. For $\mu, \nu \in \mathcal{W C}$ omp we say that $\mu \leq_{\text {dom }} \nu$ in dominance order if $\mu(1)+\mu(2)+\cdots+\mu(i) \leq \nu(1)+\nu(2)+\cdots+\nu(i)$ for all $i$. For example $(1,1,4,2) \leq_{\text {dom }}$ $(1,2,3,3)$ since $1 \leq 1,1+1 \leq 1+2,1+1+4 \leq 1+2+3$ and $1+1+4+2 \leq 1+2+3+3$. For $\mu, \nu \in \mathcal{W C O m p}$ for some $n$, such that $\mu \leq_{\text {dom }} \nu$, the dominance difference $\nu \backslash$ dom $\mu$ of $\nu$ and $\mu$ is the weak composition of length $\ell(\nu)-1$ defined $(\nu \backslash \operatorname{dom} \mu)(i):=(\nu(1)+\cdots+\nu(i))-$ $(\mu(1)+\cdots+\mu(i))$ for $i=1, \ldots, \ell(\nu)-1$. We also consider a natural operation in $\mathcal{W}$ Comp. For $\mu, \nu \in \mathcal{W C}$ omp we let $\mu \oplus \nu$ be the weak composition formed by the concatenation of $\mu$ and $\nu$. For example, $(3,2,1) \oplus(1,5,2,2)=(3,2,1,1,5,2,2)$. Sometimes we will use the notation $\mu^{+i}$ or $\mu^{-i}$ to denote the weak composition obtained from $\mu$ by adding or substracting $i$ to the last part of $\mu$ respectively.

2.2. Catalan objects. The Catalan numbers constitute one of the most important sequences in combinatorics:

$$
1,1,2,5,14,42,132,429,1430,4862,16796,58786, \ldots
$$

The $n$-th term of this sequence is commonly denoted by $C_{n}$. It is given by the simple formula $C_{n}=\frac{1}{n+1}\left(\begin{array}{c}2 n \\ n\end{array}\right)=\frac{1}{2 n+1}\left(\begin{array}{c}2 n+1 \\ n\end{array}\right)=\frac{(2 n) !}{(n+1) ! n !}$, and is completely determined by the fundamental recurrence

$$
C_{n+1}=\sum_{k=0}^{n} C_{k} C_{n-k}, \quad C_{0}=1 .
$$

The Catalan numbers are known to count a great variety of objects in mathematics [35]. Among the most remarkable ones are:

(1) planar binary trees with $n$ internal nodes;

(2) Dyck paths in an $n \times n$ grid;

(3) 312-avoiding permutations of $[n]$;

(4) noncrossing partitions of $[n]$;

(5) noncrossing matchings of $[2 n]$; 
(6) triangulations of an $(n+2)-$ gon;

(7) parenthesizations of $n+1$ consecutive letters.

Some of these families of combinatorial objects have been generalized in various directions. This includes families that are counted by the Fuss-Catalan numbers $\frac{1}{m n+1}\left(\begin{array}{c}(m+1) n \\ n\end{array}\right)$, or more generally, by the rational Catalan numbers $C_{a, b}=\frac{1}{a+b}\left(\begin{array}{c}a+b \\ a\end{array}\right)$.

The main purpose of this paper is to start a systematic study of a wider generalization indexed by a composition $s=\left(s_{1}, s_{2}, \ldots, s_{a}\right)$. The members of these generalized families will be referred to as $s$-Catalan objects.

2.3. $s$-Catalan objects. The $s$-Catalan objects corresponding to the Catalan families mentioned above are described very naturally in terms of any composition $s$, which encodes certain combinatorial information. For instance, planar binary trees can be generalized to arbitrary planar rooted trees, where the signature encodes the number of children of each internal node of the tree when read in certain order (preorder). Another example is that of Dyck paths, which can be generalized to lattice paths that lie weakly above a certain ribbon shape determined by $s$. The precise definitions and motivations for the generalized $s$-Catalan families will be presented in the coming sections. As an insight, some examples of the resulting combinatorial objects are illustrated in Figure 3.

As we shall see, these generalized families have the same intrinsic combinatorial structure (Sections 3 and 4). They are all counted by the same determinantal formula (Section 5), and are determined by the same fundamental recurrence of $s$-Catalan structures:

$$
C_{s}=\sum_{(s)} C_{s_{1}} C_{s_{2}} \cdots C_{s_{s(1)}}
$$

where the sum is over all sequences $\left(s_{1}, s_{2}, \ldots, s_{s(1)}\right)$ of compositions such that $s=(s(1)) \oplus$ $s_{1} \oplus s_{2} \oplus \cdots \oplus s_{s(1)}$ and where $C_{\emptyset}=1$ (see Section 4 ).

The classical Catalan and Fuss-Catalan combinatorics can be recovered when $s=$ $(2,2, \ldots, 2)$ and $s=(k+1, k+1, \ldots, k+1)$ respectively. The rational Catalan combinatorics corresponds to the signature $s$, where $s(i)$ is the number of boxes in row $i$ in a $b \times a$ grid that are crossed by the main diagonal.

One of our main results is to show that the generalized $s$-Catalan families are bijectively equivalent (see Figure 2).

Theorem 2.1. The following generalized familes are in bijective correspondence:

(1) s-trees;

(2) s-Dyck paths;

(3) 312-avoiding Stirling $(s-\mathbf{1})$-permutations;

(4) noncrossing $(s-\mathbf{1})$-partitions;

(5) Complete noncrossing s-matchings;

(6) s-angulations of an $(|s|-\ell(s)+2)$-gon;

(7) s-parenthesizations;

In items (3) and (4) it is assumed that $s(i) \geq 2$ for all $i$. 


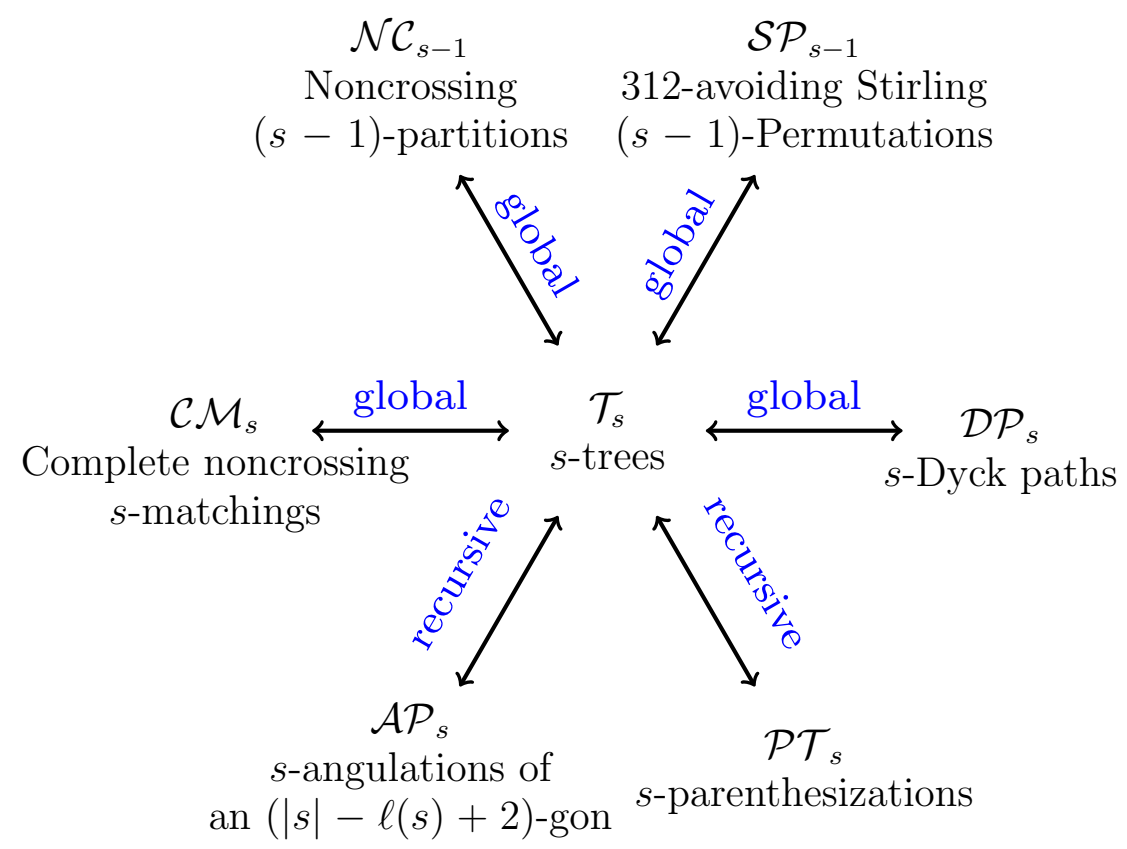

Figure 2. Bijections between $s$-Catalan objects.

The bijections between $s$-trees (1) and items (2)-(5) are presented in Section 3. The idea behind these bijections is always essentially the same: we assign some labels to the tree and then read them in some order (preorder). Figure 4 presents a quick descriptive illustration. We include detailed proofs explaining facts about these bijections for completeness, but some readers may like to skip some of the proofs and convince themselves from the description in Figure 4. The bijections between $s$-trees (1) and items (6)-(7) are explained using a fundamental recurrence in Section 4. Bijections with $s$-Dyck paths are explained in the Appendix A, see Figure 30 for a descriptive illustration.

\section{The $s$-CATAlan ZOo AND BiJECtions}

3.1. Planar rooted trees. A tree is a graph that has no loops or cycles. We say that a tree is rooted if one of its nodes is specially marked and called the root. For two nodes $x$ and $y$ on a rooted tree $T, x$ is said to be the parent of $y$ (and $y$ the child of $x$ ) if $x$ is the node that follows $y$ in the unique path from $y$ to the root. We call $y$ a descendant of $x$ if $x$ belongs to the unique path from $y$ to the root. A node is called a leaf if it has no children, otherwise is said to be internal. The degree $\operatorname{deg}(x)$ of a node $x$ in a rooted tree $T$ is defined 


\section{The s-Catalan Zoo}

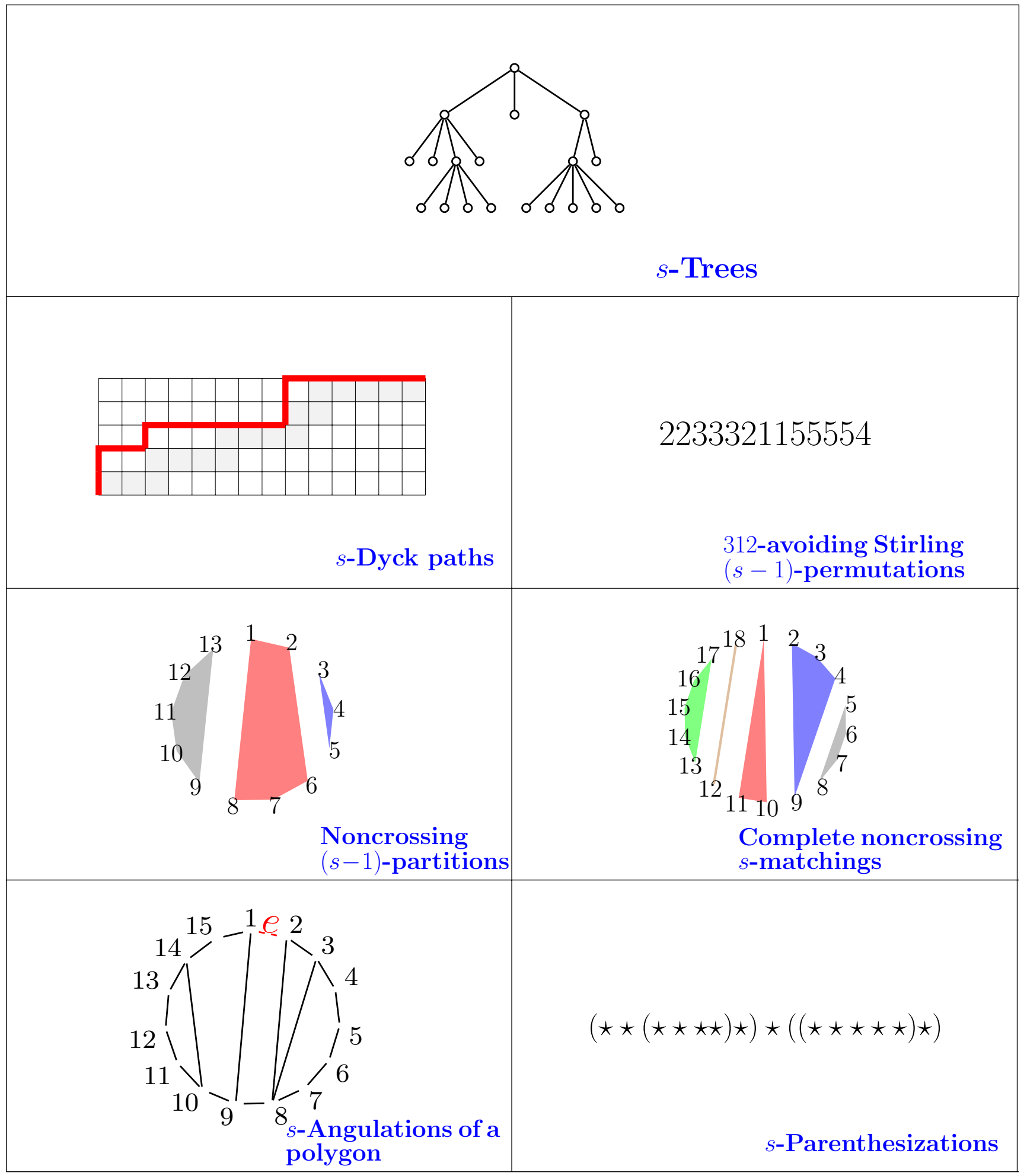

Figure 3. Examples of $s$-Catalan objects for $s=(3,4,4,2,5)$. 


\section{Bijections with $s$-Trees}

E leaves
Read labels in preorder
N internal nodes

Figure 4. Examples of the bijections with $s$-trees. 
to be the cardinality of the set of children of $x$. A rooted tree $T$ is said to be planar if for every internal node $x$ of $T$ the set of children of $x$ is totally ordered. For $n \geq 1$ we denote by $\mathcal{T}_{n}$ the set of all planar rooted trees with $n$ internal nodes and by $\mathcal{T}$ the set of all planar rooted trees. There is a unique tree $\bullet$ that has only one node that is at the same time its leaf and its root, we call this tree the identity tree. For trees $T_{1}, T_{2}, \ldots, T_{k} \in \mathcal{T}$ with roots $r_{1}, r_{2}, \ldots, r_{k}$ respectively, we denote by $\left[T_{1}, T_{2}, \ldots, T_{k}\right]$ the tree in $\mathcal{T}$ constructed by adding a new root $r$ and attaching the trees $T_{1}, T_{2}, \ldots, T_{k}$ to $r$ in a way that the order of the children of $r$ is given by $r_{i}$ being the $i$ th children for $i \in[k]$ (see Figure 5). We are going to fix an order in which to read the nodes of a tree $T=\left[T_{1}, T_{2}, \ldots, T_{k}\right] \in \mathcal{T}$. Let $r$ be the root of $\mathcal{T}$, we say that $T$ is read or traversed in preorder if we visit first the root $r$ and then we visit in preorder each of the trees $T_{i}$ in a way that $T_{i}$ is traversed before $T_{j}$ if $i<j$ for $i, j \in[k]$.

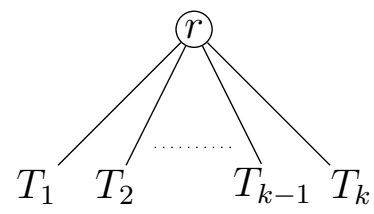

FiguRE 5. Example of $\left[T_{1}, T_{2}, \ldots, T_{k}\right]$

Let $v_{1}, v_{2}, \ldots, v_{a}$ be the internal nodes of $T$ listed in preorder, we define the signature of $T$ to be the composition

$$
\operatorname{signat}(T):=\left(\operatorname{deg}\left(v_{1}\right), \operatorname{deg}\left(v_{2}\right), \ldots, \operatorname{deg}\left(v_{a}\right)\right) .
$$

The signature of the identity tree is defined as the empty composition, i.e., $\operatorname{signat}(\bullet):=\emptyset$.

Definition 3.1 ( $s$-trees). For any $s \in \mathcal{C}$ omp, an $s$-tree is a planar rooted tree with signature $s$. We denote by

$$
\mathcal{T}_{s}=\{T \in \mathcal{T} \mid \operatorname{signat}(T)=s\}
$$

the set of $s$-trees.

Figure 6 illustrates an $s$-tree $T$ with signature $s=(3,4,4,2,5)$.

3.2. Dyck paths. We consider lattice paths in $\mathbb{N} \times \mathbb{N}$ starting at $(0,0)$ and taking only east steps $\mathrm{E}$ (in the direction $(1,0)$ ) and north steps $\mathrm{N}$ (in the direction $(0,1)$ ). For positive relatively prime integers $a$ and $b$, a rational Dyck path or an $(a, b)$-Dyck path is a lattice path with endpoint $(b, a)$ wich stays above the diagonal $y=\frac{a}{b} x$. See Figure 7 for an example of a rational Dyck path with $(a, b)=(5,8)$.

Rational Dyck paths were introduced by Armstrong, Rhoades and Williams in [3] where they started the systematic combinatorial study of rational Catalan combinatorics. This definition has as special cases the classical Dyck paths that occur for $n \geq 1$ when $a=n$ and $b=n+1$ and more generally the Fuss-Catalan generalization of Dyck paths that occur when $a=n$ and $b=k n+1$ for a fixed $k \in \mathbb{P}$.

Note that in the $b \times a$ grid the diagonal $y=\frac{a}{b} x$ crosses certains cells. Those cells define a ribbon shape that cannot be crossed by any rational Dyck path in order to stay above 


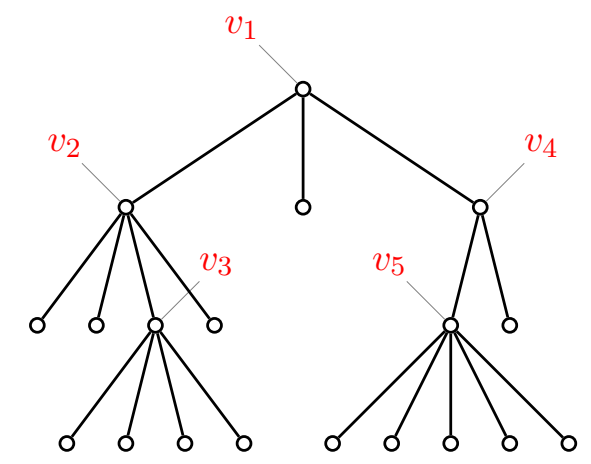

Figure 6. Example of a tree in $\mathcal{T}_{(3,4,4,2,5)}$

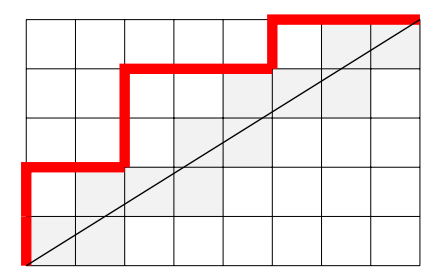

Figure 7. Example of a $(5,8)$ rational path

the diagonal (see the gray shape in Figure 7). Let $s \in \mathcal{C}$ omp be defined such that $s(i)$ is the number of cells in row $i$ crossed by the diagonal for each $i \in[a]$, we call $s$ the degree sequence associated to the pair $(a, b)$. For any rational pair $(a, b)$ with residue $r=b-\left\lfloor\frac{b}{a}\right\rfloor a$ it is not difficult to check that the degree sequence is given by

$$
s(i)=\left\lceil\frac{b}{a}\right\rceil+\chi(0<i r(\bmod \mathrm{a})<r)
$$

where $\lfloor x\rfloor$ and $\lceil x\rceil$ are the floor and ceiling functions respectively, and $\chi$ is the function

$$
\chi(S)=\left\{\begin{array}{lc}
1: & S \text { is true } \\
0: & \text { otherwise }
\end{array}\right.
$$

For example for $(5,8)$ the degree sequence is $(2,3,2,3,2)$ since $\left\lceil\frac{8}{5}\right\rceil=2, r=8-\left\lfloor\frac{8}{5}\right\rfloor 5=3$ and $3(1,2,3,4,5)=(3,1,4,2,0)(\bmod 5)($ see Figure 7$)$.

Every lattice path can be encoded with a string of the letters $N$ and $E$ representing the north steps and east steps respectively, where the reading order of the path starts at the origin $(0,0)$. For example the path $D$ of Figure 7 can be represented as $D=$ $N N E E N N E E E N E E E$. If the lattice path is a rational Dyck path then it must have exactly $a$ north steps and it must start with a north step and finish with an east step, so it is of the form $D=N E^{i_{1}} N E^{i_{2}} \cdots N E^{i_{a}} E$ where $E^{i}$ indicates a string of $i$ occurrences of the letter $E$ and then it can be uniquely associated with the weak composition $\mu(D):=$ $\left(i_{1}, i_{2}, \ldots, i_{a}\right)$. So we can think about $(a, b)$-Dyck paths as weak compositions of $b-1$ of length $a$. For the example in Figure 7 we have that $\mu(D)=(0,2,0,3,2)$ (note that the last $E$ is not counted). There is one special rational Dyck path, the identity path $D^{\emptyset}=E$, 


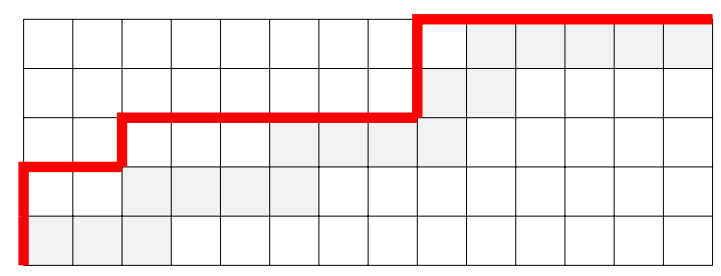

Figure 8. Example of a $(3,4,4,2,5)$-Dyck path

with exactly one east step and without any north steps. By convention, this is considered as the unique $(0,1)$-Dyck path. We also have that $\mu\left(D^{\emptyset}\right)=\emptyset$ (the empty weak composition). It is not hard to see that in dominance order there is a maximal $(a, b)$-Dyck path $\mathcal{D}$ that is defined by the boundary of the forbidden ribbon shape. This path has $\mu(\mathcal{D})=s-\mathbf{1}:=$ $(s(1)-1, s(2)-1, \ldots, s(a)-1)$. An equivalent definition of a rational Dyck path is a path $D$ such that $\mu(D) \leq_{\text {dom }} \mu(\mathcal{D})=s-\mathbf{1}$.

There is really nothing special about the rational ribbon shape apart from the fact that it is defined using the diagonal of a $b \times a$ rectangle. The information of the numbers $a$ and $b$ can be obtained from the degree sequence $s$. Indeed, $a=\ell(s)$ and $b=|s|-\ell(s)+1$.

Definition 3.2 (s-Dyck paths). For any $s \in \mathcal{C}$ omp, that we will call a signature, we define an $s$-Dyck path $D$ to be a lattice path starting at $(0,0)$ and ending at $(|s|-\ell(s)+1, \ell(s))$ such that $\mu(D) \leq_{\text {dom }} s-\mathbf{1}$. We denote by $\mathcal{D} \mathcal{P}_{s}$ the set of $s$-Dyck paths, and refer to an $s$-Dyck path as a Dyck path when $s$ is clear from the context.

An example of a $(3,4,4,2,5)$-Dyck path $D$ with $\mu(D)=(0,2,6,0,5)$ is illustrated in Figure 8. We will say that a signature $s$ is rational when $s$ is obtained as in equation 3.1 for a pair $(a, b)$ of relatively prime numbers.

We define the area vector of an $s$-Dyck path $D$ to be the weak composition $\operatorname{Area}(D):=$ $(0) \oplus(s-\mathbf{1}) \backslash_{\text {dom }} \mu(D)$. Its entries are the number of boxes, counted by rows, between $D$ and the ribbon shape determined by $s$. The area of $D$ is area $(D):=|\operatorname{Area}(D)|$. In the example of Figure 8 Area $(D)=(0,2,3,0,1)$ and area $(D)=5$.

3.2.1. Bijection between $s$-trees and $s$-Dyck paths. Let $T$ be an $s$-tree. Label the internal nodes of $T$ with $N$ 's and the leaves with $E$ 's. The lattice path $\xi(T)$ associated to $T$ is obtained by reading the sequence of $N$ 's and $E$ 's on the nodes of $T$ in preorder. The function $\xi$ maps the identity tree $\bullet$ to the identity path $E$. An example of this bijection is illustrated in Figure 9.

We define the area labeling of a tree as the labeling of the nodes such that the root is labeled by 0 and the $i$ th child of an internal node $x$, from right to left, is labeled by $i-1$ plus the label of $x$. This is illustrated for our example in Figure 10. It turns out that the area vector of $D=\xi(T)$ can be read directly from $T$.

Lemma 3.3. The entries of the area vector of the Dyck path $D=\xi(T)$ are the labels in the area labeling of $T$ of the internal nodes listed in preorder.

Proof. The root of the tree corresponds to the first north step of the path, which contributes a zero entry in the area vector. Let $x$ and $y$ be two internal nodes of $T$ such that $y$ is 


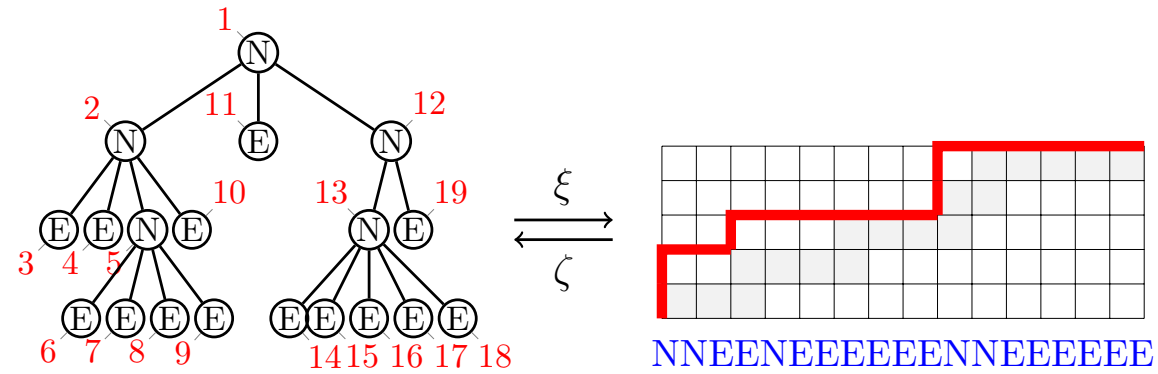

FigurE 9. Example of the bijection between $s$-trees and $s$-Dyck paths.

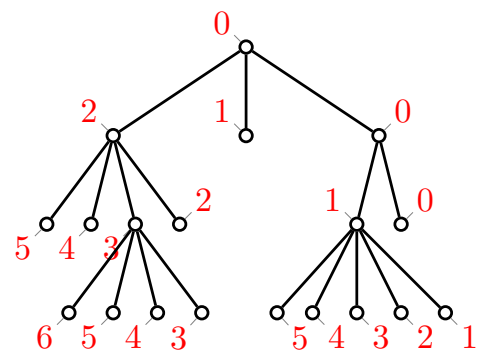

FigurE 10. The area labeling of a tree $T$. The area vector of $D=\xi(T)$ is the sequence of labels of the internal nodes listed preorder.

the $i$ th child from right to left of $x$. Let $a(x)$ and $a(y)$ be the entries of the area vector of $D$ of the corresponding north steps in $D$. We need to show that $a(y)=a(x)+i-1$. Let $u_{1}, \ldots, u_{j}$ be the internal nodes strictly between $x$ and $y$ in $T$ in preorder, and denote $u_{0}=x$. Then,

$$
a(y)=a(x)+\sum_{k=0}^{j}\left(\operatorname{deg}\left(u_{k}\right)-1\right)-E(x, y),
$$

where the sum of the degrees minus one is the increase of area determined by the ribbon shape and $E(x, y)$ denotes the number of east steps (or leafs) between $x$ and $y$. Since $E(x, y)=\sum_{k=0}^{j} \operatorname{deg}\left(u_{k}\right)-j-i$, then $a(y)=a(x)-(j+1)+j+i=a(x)+i-1$ as desired.

Lemma 3.4. The path $\xi(T)$ is an s-Dyck path.

Proof. By definition, all the entries of the area labeling of the tree $T$ are nonnegative. Lemma 3.3 then implies that the area vector of $\xi(T)$ has nonnegative entries and therefore that it is an $s$-Dyck path.

We will see below that the map $\xi$ is actually a bijection between $s$-trees and $s$-Dyck paths. The inverse of this map is described below. Let $D$ be an $s$-Dyck path and $\mu=\mu(D)$. Define the tree $\zeta(D)$ associated to $D$ recursively as follows.

(1) First, start with a root $r=v_{1}$ with $s(1)$ children. 
(2) Attach an internal node $v_{2}$ with $s(2)$ children at the $(\mu(1)+1)$ th leaf, ordered from left to right.

(3) In general, at the $i$ th step of the process we have a tree $\tau_{i}$ with internal nodes $v_{1}, \ldots, v_{i}$ in preorder. The $(i+1)$ th tree $\tau_{i+1}$ is obtained from $\tau_{i}$ by attaching an internal node $v_{i+1}$ with $s(i+1)$ children at the $(\mu(i)+1)$ th leaf that appear after $v_{i} \in \tau_{i}$ in preorder.

(4) The process finishes after attaching the $a$ internal nodes $v_{1}, \ldots, v_{a}$, where $a=\ell(s)$.
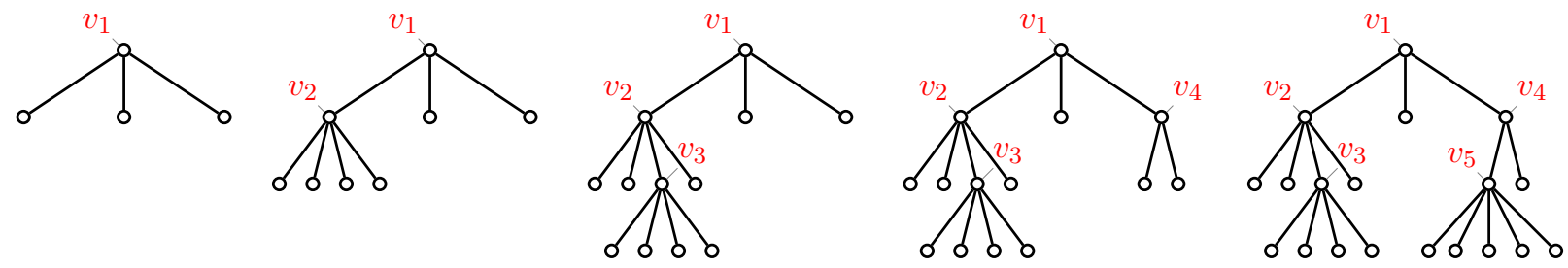

FiguRE 11. The recursive construction of the tree $\zeta(D)$ for the Dyck path with signature $s(D)=(3,4,4,2,5)$ and composition $\mu(D)=(0,2,6,0,6)$.

Lemma 3.5. Let $D$ be an s-Dyck path. The map $\zeta$ is well defined and $\zeta(D)$ is an s-tree.

Proof. In order to show that the map $\zeta$ is well defined we need to show that for $i<a$ there are at least $\mu(i)+1$ leafs in the tree $\tau_{i}$ that appear after the internal node $v_{i} \in \tau_{i}$ in preorder. This guarantees that a new node $v_{i+1}$ can be added at the right place and that the tree $\tau_{i+1}$ in the recursive definition can be constructed. For $i=1$ this is clear since $v_{1}$ has $s(1) \geq \mu(1)+1$ children. In general, since $D$ is a path that lies above the ribbon shape defined by $s$, for $i<a$ we have

$$
\mu(1)+\cdots+\mu(i) \leq(s(1)-1)+\cdots+(s(i)-1) .
$$

The total number of leafs in $\tau_{i}$ is equal to $\sum_{k=1}^{i}(s(k)-1)+1$, from which $\sum_{k=1}^{i-1} \mu(k)$ are before $v_{i}$ in preorder. Combining these two equations with the inequality (3.2) we obtain that the number of leafs in $\tau_{i}$ after $v_{i}$ in preorder is

$$
\sum_{k=1}^{i}(s(k)-1)+1-\sum_{k=1}^{i-1} \mu(k) \geq \mu(i)+1 .
$$

This shows that the map is well defined. The signature of the tree $\zeta(D)$ is by construction equal to $s=(s(1), \ldots, s(a))$.

Theorem 3.6. The map $\xi: \mathcal{T}_{s} \rightarrow \mathcal{D P}_{s}$ is a bijection between s-trees and s-Dyck paths. The inverse of $\xi$ is $\zeta$.

Proof. We will show that $D=\xi(T)$ if and only if $T=\zeta(D)$. This implies that both maps are bijections and that they are inverses to each other. 
Let $D=\xi(T)$ and $\mu=\mu(D)$. Denote by $v_{1}, \ldots, v_{a}$ the internal nodes of $T$ in preorder. By the definition of $\xi$, there are exactly $\mu(i)$ leafs between $v_{i}$ and $v_{i+1}$ traveling $T$ in preorder. This condition completely characterizes $T$ and is satisfied by $\zeta(D)$. Therefore, $T=\zeta(D)$.

On the other hand, if $T=\zeta(D)$ then $T$ is the unique tree with internal nodes $v_{1}, \ldots, v_{a}$ in preorder such that there are exactly $\mu(i)$ leafs between $v_{i}$ and $v_{i+1}$, for $i<a$. Therefore, $D=\xi(T)$.

3.3. 312-avoiding Stirling permutations. Let $s=(s(1), s(2), \ldots, s(a))$ be a composition. An s-permutation is any multiset permutation (or multipermutation) of the multiset $\left\{1^{s(1)}, 2^{s(2)}, \ldots, a^{s(a)}\right\}$, where there are $s(i)$ copies of each $i$. Let us denote the set of $s$ permutations as $\mathfrak{S}_{s}$, then we have that

$$
\left|\mathfrak{S}_{s}\right|=\left(\begin{array}{c}
|s| \\
s
\end{array}\right):=\frac{|s| !}{s(1) ! s(2) ! \cdots s(a) !} .
$$

For multipermutations $\tau$ and $\sigma$ we say that $\sigma$ contains the pattern $\tau$ if there is a subword of $\sigma$ where the elements have the same relative order as the elements in $\tau$. For example, the multipermutation $\sigma=1132235544$ contains the pattern $\tau=212$ because the elements in the subword 323 of $\sigma$ are in the same relative order than the elements in $\tau$. A multipermutation can contain many patterns and multiple or no occurrences of a particular pattern. For example $\sigma=1132235544$ contains no occurrence of the pattern 321. We say that $\sigma$ avoids $\tau$ or is $\tau$-avoiding if it does not contain any occurrence of the pattern $\tau$.

A particular example of pattern avoiding multipermutations are the 212-avoiding $s$ permutations introduced by Gessel and Stanley in [10] when they studied a generalization of Euler's formula for Eulerian polynomials. They considered the descent enumerator of 212-avoiding multipermutations of $\{1,1,2,2, \cdots, n, n\}$, i.e., multipermutations in which all the numbers between the two occurrences of any fixed number $m$ are larger than $m$. To this family, belongs for example the permutation 12234431 but not the permutation 11322344 since 2 is less than 3 and 2 is between the two occurrences of 3 . They called this family of multipermutations Stirling Permutations and they have been studied by many other authors, see for example [17, 13, 27, 26, 25, 11, 6, 32].

The set of $s$-permutations have also already appeared in the work on Hopf algebras of Novelli and Thibon [24]. They study generalizations of the Malvenuto-Reutenauer Hopf algebra of permutations [23] to multipermutations of $\left\{1^{m}, 2^{m}, \ldots, n^{m}\right\}$, and of the LodayRonco Hopf algebra of planar binary trees $[20]$ to $(m+1)$-ary trees. The authors introduce a notion of metasylvester congruence on permutations that allows them to obtain Hopf algebras based on decreasing trees. The element representatives of the congruence classes are 121-avoiding s-permutations for $s=(m, m, \ldots, m)$, see [24, Proposition 3.10]. These 121-avoiding $s$-permutations and associated lattices called metasylvester lattices are also studied by Pons in [30], where they are also related to the combinatorics of $m$-Tamari lattices introduced by F. Bergeron.

Definition 3.7 (312-avoiding Stirling $s$-permutations). A Stirling $s$-permutation is a multipermutation of $\left\{1^{s(1)}, 2^{s(2)}, \ldots, a^{s(a)}\right\}$ that avoids the pattern 212. A 312-avoiding Stirling $s$-permutation is a multipermutation of the same multiset that avoids both patterns 212 
and 312. We denote by $\mathcal{S} \mathcal{P}_{s}$ the set of Stirling $s$-permutations, and by $\mathcal{S} \mathcal{P}_{s}(312)$ the set of 312-avoiding Stirling $s$-permutations.

For instance, 1223321 and 2332211 are 312-avoiding Stirling (2,3,2)-permutations, while 3312221 is a Stirling $(2,3,2)$-permutation that is not 312-avoiding. See Figure 12 for the complete example of $\mathcal{S P}(2,3,2)$ and $\mathcal{S P}(2,3,2)(312)$.

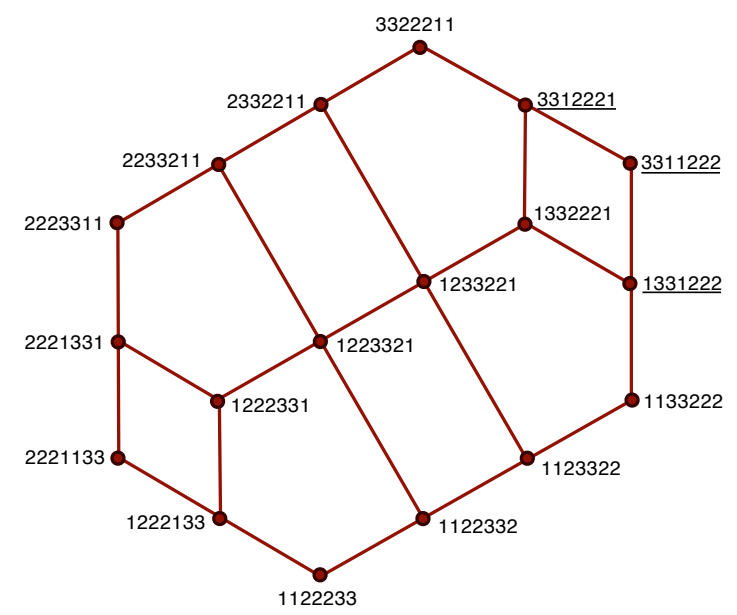

Figure 12. All Stirling $s$-permutations for $s=(2,3,2)$ (drawn as the elements of a lattice similarly as in [30]). The 312-avoiding are the nonunderlined ones. There are 15 of them, which are in bijection with the 15 $(3,4,3)$-trees.

Remark 3.8. Replacing the numbers from 1 to $a$ for the numbers from $a$ to 1 respectively and reversing the permutation transforms bijectively $(212,312)$-avoiding $s$-permutations into (121, 231)-avoiding $\overleftarrow{s}$-permutations, where $\overleftarrow{s}=(s(a), \ldots, s(1))$ denotes the composition obtained by reading $s$ in reverse order. This family of $(121,231)$-avoiding $\overleftarrow{s}$ permutations is a generalization of 231-avoiding permutations which is also an $s$-Catalan family.

3.3.1. Bijection between s-increasing trees and Stirling $(s-\mathbf{1})$-permutations. An increasing tree is a planar rooted tree whose internal nodes have been decorated with the elements of $[a]$ (where $a$ is the number of internal nodes) such that any path of internal nodes away from the root has strictly increasing labels. An $s$-increasing tree is an increasing tree such that the internal node with label $i$ has exactly $s(i)$ children. We denote by $\mathcal{I}_{s}$ the set of $s$-increasing trees. Figure 13 shows two examples of $(3,4,4,2,5)$-increasing trees.

Gessel provided a simple bijection between the sets $\mathcal{I T}_{s}$ and $\mathcal{S P}_{s-\mathbf{1}}$ that we describe below (Gessel's result is unpublished but can be found for example in [13]). Before explaining this bijection we need to introduce the notion of a cavern in a planar rooted tree. For a tree $T \in \mathcal{T}$ and an internal node $x$ of $T$ we call a cavern of $T$ dermined by $x$ to the 

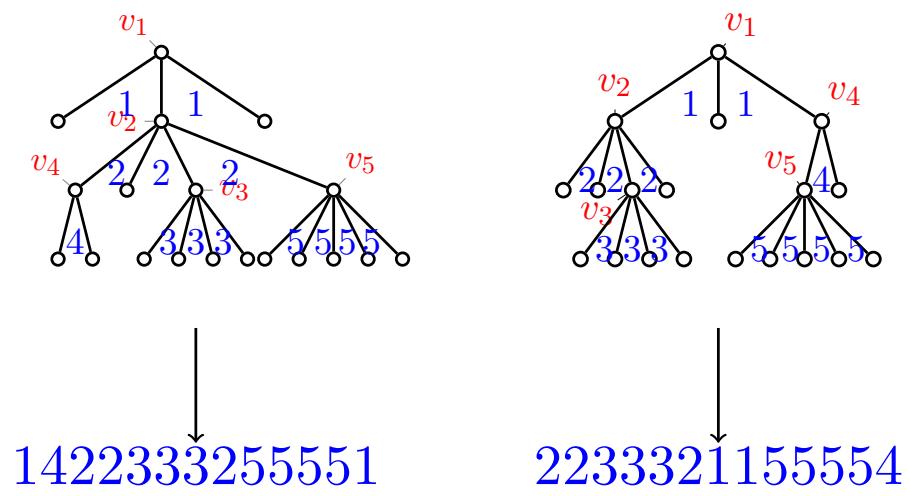

FiguRE 13. Example of the map $\Sigma: \mathcal{I T}_{s} \rightarrow \mathcal{S P}_{s-1}$

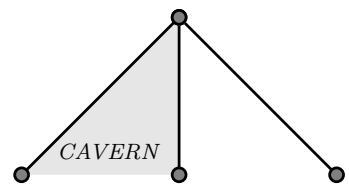

Figure 14. A cavern

space between any two consecutive children of $x$ (see Figure 14), i.e., if the ordered set of children of $v$ is $\left\{c_{1}, c_{2}, \ldots, c_{k}\right\}$ then the set of caverns determined by $v$ is

$$
\operatorname{caverns}(v)=\left\{\left\{c_{1}, c_{2}\right\},\left\{c_{2}, c_{3}\right\}, \ldots,\left\{c_{k-1}, c_{k}\right\}\right\} .
$$

We also define caverns $(T):=\cup_{i=1}^{a} \operatorname{caverns}\left(v_{a}\right)$. Any internal node $v$ of $\operatorname{deg}(v)=k$ defines exactly $k-1$ caverns and hence there are in total $|\operatorname{caverns}(T)|=\sum_{i=1}^{a} \operatorname{deg}\left(v_{i}\right)-a$ caverns in a tree $T \in \mathcal{T}$ with $a$ internal nodes.

The set of caverns has a very natural ordering. We can associate each cavern $C=$ $\left\{c_{i}, c_{i+1}\right\}$ of $x$ to the largest children $c_{i+1}$ of $x$ in $C$. In that way we can think of the caverns as the set of nonminimal children of internal nodes in $T$ (being $c_{1}$ the minimal child of $x$ ). The order in the set caverns $(T)$ is the one induced by preorder in the set of nonminimal children in $T$. (see Figure 18). We refer to this order as the preorder order of the caverns of $T$.

For $T \in \mathcal{I T}_{s}$ we label all the caverns with the label of its corresponding internal node. Reading the caverns in preorder gives rise to an $(s-\mathbf{1})$-permutation $\Sigma(T)$. Since $T$ is an increasing tree, the caverns with label $i$ are read before or after all the caverns with label $j$ for $i<j$. So, the resulting $(s-\mathbf{1})$-permutation is 212-avoiding, so a Stirling permutation.

The process described above maps an increasing tree $T \in \mathcal{I T}_{s}$ to a $\operatorname{Stirling}(s-\mathbf{1})$ permutation $\Sigma(T)$. In fact, every Stirling $(s-\mathbf{1})$-permutation can be attained uniquely by exactly one such a tree. We construct the inverse map as follows: let $\sigma$ be a Stirling $(s-\mathbf{1})$ permutation. Put a root labeled 1 with $s(1)$ children labelled with the $s(1)$ permutations that are separated by the ones in $\sigma$ (some of which might be empty). Repeat the process with each of the non-empty permutations appearing in the process with the smallest value 
in the permutation instead of the one. The resulting tree $\Lambda(\sigma)$ at the end of the process is the desired increasing tree, see Figure 15 for an example.

2233321155554

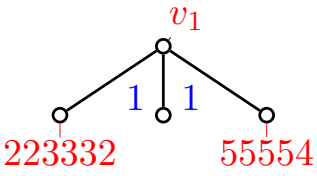

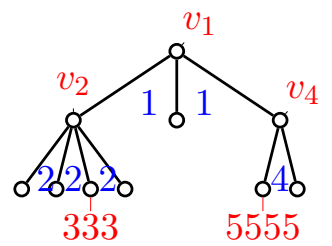

FiguRE 15. Example of the map $\Lambda: \mathcal{S P}_{s-\mathbf{1}} \rightarrow \mathcal{I}_{s}$

Proposition 3.9 (Gessel c.f. [13]). The map $\Sigma: \mathcal{I T}_{s} \rightarrow \mathcal{S P}_{s-1}$ is a bijection between increasing trees and Stirling $(s-\mathbf{1})$-permutations. The inverse of $\Sigma$ is $\Lambda$.

3.3.2. Bijection between s-trees and 312-avoiding Stirling $(s-\mathbf{1})$-permutations. Note that if we decorate the internal nodes of an $s$-tree $T$ with the values of $[a]$ in preorder we obtain an increasing tree $\tilde{T} \in \mathcal{I} \mathcal{T}_{s}$, hence there is an injection $\mathcal{T}_{s} \hookrightarrow \mathcal{I} \mathcal{T}_{s}$. It turns out that the image of the composition $\mathcal{T}_{s} \hookrightarrow \mathcal{I} \mathcal{T}_{s} \rightarrow \mathcal{S P} \mathcal{P}_{s-\mathbf{1}}$ is precisely the set $\mathcal{S P}_{s-\mathbf{1}}(312)$. We denote the map determined by this composition by $\tilde{\Sigma}$. We also denote by $\tilde{\Lambda}(T)$ the $s$-tree obtained by removing the labels from the increasing tree $\Lambda(T)$. The Stirling permutation 2233321155554 that appear in Figures 13 and 15 is 312-avoiding, giving an example of the discussed bijection.

Theorem 3.10. The map $\tilde{\Sigma}: \mathcal{T}_{s} \rightarrow \mathcal{S P}_{s-1}(312)$ is a bijection between s-trees and 312 avoiding Stirling $(s-\mathbf{1})$-permutations. The inverse of $\tilde{\Sigma}$ is $\tilde{\Lambda}$.

Proof. It remains to show:

(1) For any $s$-tree $T$, its image $\tilde{\Sigma}(T) \in \mathcal{S P}_{s-\mathbf{1}}(312)$.

(2) For any $\sigma \in \mathcal{S P}_{s-\mathbf{1}}(312)$, the tree $\tilde{\Lambda}(\sigma)$ is an $s$-tree.

Proof of (1).Label the internal nodes of an $s$-tree $T$ in preorder, and the caverns with the label of its corresponding internal node. By Proposition 3.9 we know that reading the caverns in preorder gives rise to a Stirling $(s-\mathbf{1})$-permutation $\Sigma(T)$. Moreover, we will see next that it is also 312-avoiding. We proceed by contradiction; assume the permutation has a subsequence $k \ldots i \ldots j$ with $i<j<k$. The internal node $k$ (labeled in preorder) necessarily has to be a descendant of the node $i$ that is not coming from its right most child. Similarly, the node $k$ is a descendant of the node $j$ that is not coming from its right most child. Drawing the unique path from node $k$ to the root visits both nodes $i$ and $j$. Since $i<j$, then $j$ has to be a descendant of $i$. From this observations it is straight forward to see from the tree that no cavern $i$ appears in preorder between two caverns $k$ and $j$. Thus the pattern 312 is impossible.

Proof of (2) Let $\sigma \in \mathcal{S P}_{s-\mathbf{1}}(312)$. By Proposition 3.9, we know taht the tree $\Lambda(\sigma)$ is increasing. We need to show that the labeling of its internal nodes is given by preorder. 


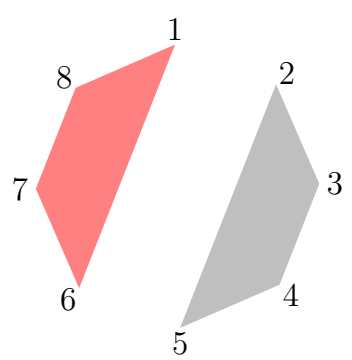

(A) Noncrossing

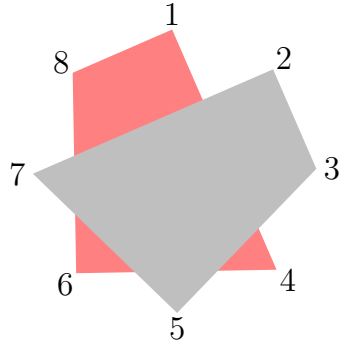

(B) Crossing

FIGURE 16. Example of noncrossing and crossing partitions of 8

We proceed by contradiction; assume that the labeling in $\Lambda(\sigma)$ violates preorder. Consider the minimal pair $v_{1}, v_{2}$ in lexicographic preorder such that $v_{1}<v_{2}$ in preorder but the label of $v_{1}$ is larger than the label of $v_{2}$. Let $v_{0}$ be the smallest common ancestor of $v_{1}$ and $v_{2}$. Since the tree is increasing, $v_{2}$ can not be a descendant of $v_{1}$. Therefore $v_{0} \neq v_{1}, v_{2}$, furthermore it has smaller label than $v_{1}$ and $v_{2}$ by lexicographic minimality of the pair $v_{1}, v_{2}$. If $r_{0}, r_{1}, r_{2}$ are the labels of $v_{0}, v_{1}, v_{2}$ respectively, then the permutation $\sigma$ corresponding to the tree contains a subsequence $r_{1}, r_{0}, r_{2}$ which satisfies $r_{0}<r_{2}<r_{1}$, and so it is not 312-avoiding.

3.4. Noncrossing partitions. A partition $\pi=\left\{\pi_{1}, \pi_{2}, \ldots, \pi_{k}\right\}$ of $[n]$ is a collection of disjoint subsets $\pi_{i} \subseteq[n]$ such that $[n]=\cup_{i} \pi_{i}$. We call each $\pi(i)$ a block of $\pi$ and denote by $|\pi|$ the number of blocks in $\pi$. We are also defining a standard order in which to read the blocks of a partition $\pi$. We read the parts in increasing order of its minimal elements, that is, $\min \left(\pi_{i}\right)<\min \left(\pi_{j}\right)$ if and only if $i<j$. We call this order the minimal order of the blocks of $\pi$.

We say that a partition $\pi$ of $[n]$ is noncrossing if there are no $x<y<z<w \in[n]$ such that $x, z \in \pi_{i}$ and $y, w \in \pi_{j}$ with $i \neq j$. See Figure 16 for an example of noncrossing and crossing partitions of 8 ; note that in Figure $16 \mathrm{~b}$ the subsets $\{3,5\}$ and $\{4,6\}$ belong to different blocks. We denote by $\mathcal{N C}_{n}$ the set of noncrossing partitions of $[n]$. In addition, let $\pi_{i}$ and $\pi_{j}$ be two blocks of $\pi$, we say that $\pi(j)$ is nested inside $\pi_{i}$ if there is a bipartition $\pi_{i}=B_{1} \cup B_{2}$ with both $B_{1}$ and $B_{2}$ nonempty such that $x \leq y \leq z$ for all $x \in B_{1}, y \in \pi_{j}$ and $z \in B_{2}$.

Definition 3.11 (Noncrossing $s$-partitions). To each partition $\pi=\left\{\pi_{1}, \pi_{2}, \ldots, \pi_{k}\right\}$ of $[n]$ (where the blocks are ordered according its minimal order) we associate the composition $\mu(\pi):=\left(\left|\pi_{1}\right|,\left|\pi_{2}\right|, \ldots,\left|\pi_{k}\right|\right)$. For $s \in \mathcal{C}$ omp, we say that $\pi$ is an $s$-partition if $s \leq \mu(\pi)$, that is, if $s$ is a refinement of $\mu(\pi)$. We denote the set of noncrossing $s$-partitions by $\mathcal{N} \mathcal{C}_{s}$.

For example, $\mathcal{N C}_{\left(1^{n}\right)}$ is the set of noncrossing partitions and $\mathcal{N C}\left(k^{n}\right)$ is the set of $k$-divisible noncrossing partitions of $[n k]$, that is, the set of noncrossing partitions of $[n k]$ whose blocksizes are all divisible by $k$. The noncrossing partition in Figure 17 is a $(2,3,3,1,4)$-partition, $(1,1,2,1,3,5)$-partition, (13)-partition and so on; but for example is not a $(3,3,3,4)$ partition since $(5,3,5)$ is not refined by $(3,3,3,4)$. 


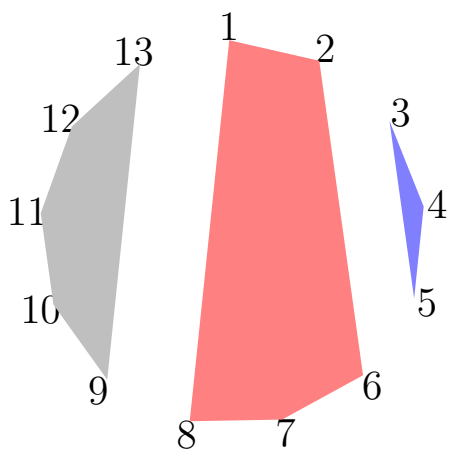

Figure 17. Example of a noncrossing $(2,3,3,1,4)$-partition
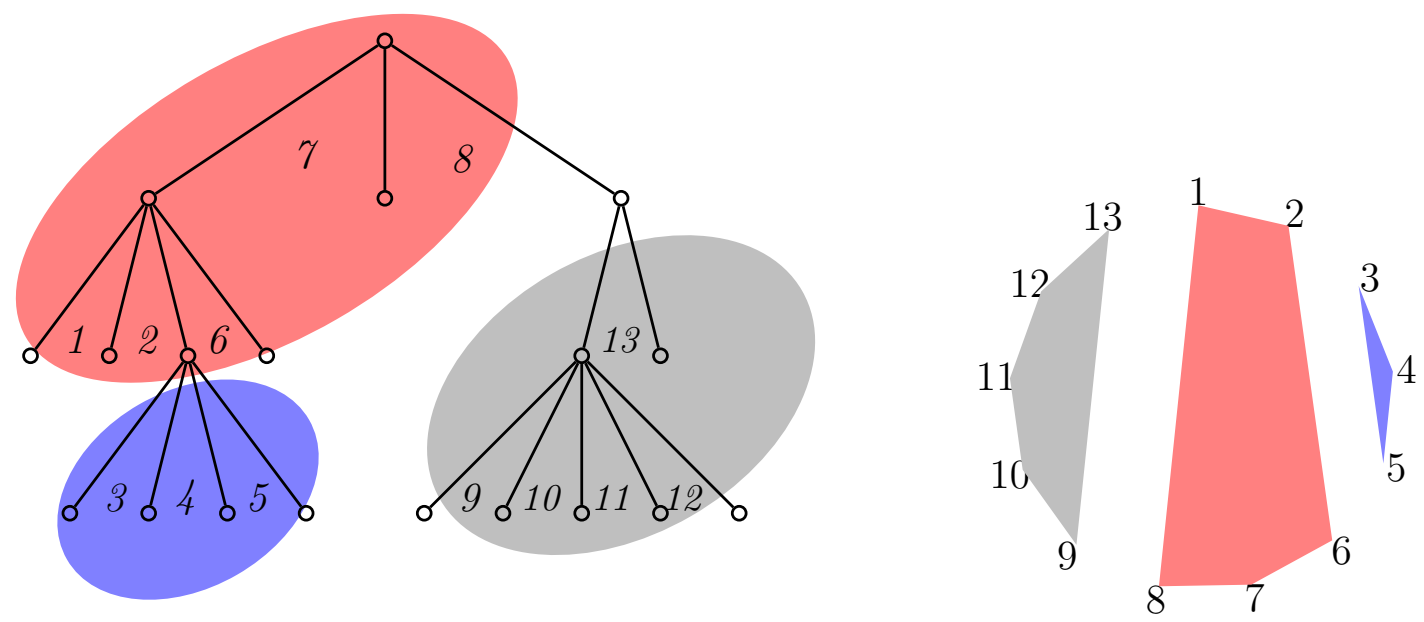

FiguRE 18. Example of the bijection between $s$-trees and noncrossing $(s-$ 1)-partitions. The caverns of the tree are ordered in preorder.

3.4.1. Bijection between $s$-trees and noncrossing $(s-\mathbf{1})$-partitions. Let $s \in \mathcal{C}$ omp be such that $s_{i} \geq 2$ for all $i$ and recall that $s-\mathbf{1}:=(s(1)-1, s(2)-1, \ldots, s(\ell(s))-1)$. We also define $\emptyset-1:=\emptyset$. We will define a bijection $\phi: \mathcal{T}_{s} \rightarrow \mathcal{N C}_{s-1}$ by labeling the caverns of the tree in preorder and grouping the labels according to certain rule.

Recall that given two nodes $x$ and $y$ of $T, y$ is called a descendant of $x$ if $x$ belongs to the unique path from $y$ to the root. A node $y$ is called a left descendant of $x$ if $y$ is the minimal child of $x$ or the minimal child of a left descendant of $x$. We denote by $\operatorname{left}(x)$ the set of left descendant internal nodes of $T$ union with $\{x\}$.

Let $C_{1}, C_{2}, \ldots, C_{|s-\mathbf{1}|}$ be the preorder of the caverns of $T \in \mathcal{T}_{s}$. We define the function $\phi$ as follows: Let $v_{1}, \ldots, v_{a}$ be the internal nodes of $T$ in preorder, and let $v_{i_{1}}, \ldots, v_{i_{k}}$ be the internal nodes that are not a minimal child of another internal node. In particular, 
$v_{i_{1}}=v_{1}$ is the root of the tree. Denote by

$$
\pi_{j}:=\bigcup_{v \in \operatorname{left}\left(v_{i_{j}}\right)} \operatorname{caverns}(v),
$$

where we are identifying a cavern $C_{i}$ with its index $i$. The partition associated to $T$ is defined

$$
\phi(T):=\left\{\pi_{1}, \ldots, \pi_{k}\right\} .
$$

An example of this map is illustrated in Figure 18.

For simplicity, we denote by $\left\{B_{1}, \ldots, B_{k}\right\}$ the partition of $[a]$ determined by the indices $i_{1}, \ldots, i_{k}$ in the following way:

$$
B_{j}= \begin{cases}{\left[i_{j}, i_{j+1}\right)} & \text { for } 1 \leq j<a \\ {\left[i_{k}, a\right]} & \text { for } j=k .\end{cases}
$$

Lemma 3.12. For $1 \leq j \leq k$, the internal nodes of $\operatorname{left}\left(v_{i_{j}}\right)$ are the nodes $\left\{v_{\ell}\right\}_{\ell \in B_{j}}$.

Proof. Let $1 \leq j<k$. The set $\operatorname{left}\left(v_{i_{j}}\right)$ is obtained from $v_{i_{j}}$ by consecutively adding in preorder left minimal children whenever possible. The process stops when following preorder we find the first internal node that is not a minimal child. Since this internal node is $v_{i_{j+1}}$ then the internal nodes of $\operatorname{left}\left(v_{i_{j}}\right)$ are exactly the nodes $\left\{v_{\ell}\right\}_{\ell \in\left[i_{j}, i_{j+1}\right)}$. If $j=k$, the process does not stop until covering all remaining internal nodes of $T$, and so, the internal nodes of $\operatorname{left}\left(v_{i_{k}}\right)$ are the nodes $\left\{v_{\ell}\right\}_{\ell \in\left[i_{k}, a\right]}$.

Lemma 3.13. The partition $\phi(T)$ is a noncrossing $(s-\mathbf{1})$-partition.

Proof. To see that $\phi(T)$ is noncrossing consider $1 \leq r<s \leq k$. Since $v_{i_{r}}$ comes before $v_{i_{s}}$ in preorder we have two possibilities, either $v_{i_{s}}$ is a descendant of $v_{i_{r}}$ or they are unrelated. And note that in these two cases preorder implies that $\pi_{s}$ is either nested in $\pi_{r}$ or that all the caverns in $\pi_{r}$ come in preorder before the ones in $\pi_{s}$.

To see that $\phi(T)$ is an $(s-\mathbf{1})$-partition note that an internal node $v_{\ell}$ of $T$ has exactly $s(\ell)-1$ caverns. Lemma 3.12 then implies that $\left|\pi_{j}\right|=\sum_{\ell \in B_{j}}(s(\ell)-1)$ for $1 \leq j \leq k$. Since the sets $B_{j}$ form a partition of $[a]$ with increasing adjacent consecutive parts, then $s-\mathbf{1}$ is a refinement of $\mu(\pi)$.

Given a noncrossing $(s-\mathbf{1})$-partition $\pi=\left\{\pi_{1}<\pi_{2}<\cdots<\pi_{k}\right\}$ we will construct a tree $T=\psi(\pi)$ such that $\phi(T)=\pi$. Since $\pi$ is an $(s-\mathbf{1})$-partition we have that $\left(\left|\pi_{1}\right|,\left|\pi_{2}\right|, \ldots\left|\pi_{k}\right|\right)$ is refined by $(s-\mathbf{1})$ meaning that $s$ can be written as $s=\tilde{s}_{1} \oplus \tilde{s}_{2} \oplus \cdots \tilde{s}_{k}$ with $\left|\pi_{j}\right|=\left|\tilde{s}_{j}-\mathbf{1}\right|$.

We say that a tree is a left descendant tree if every internal node is either the root or the minimal (leftmost) child of another internal node. For $1 \leq j \leq k$, let $\tau_{j}$ be the (unique) left descendant tree with signature $\tilde{s}_{j}$, and label the caverns of $\tau_{j}$ in preorder with the elements of $\pi_{j}$ (note this is possible since the number of caverns of $\tau_{j}$ is exactly equal to $\left.\left|\pi_{j}\right|\right)$.

Lemma 3.14. Let $\left\{\tau_{1}, \ldots, \tau_{k}\right\}$ be a set of left descendant trees such that the caverns of tree $\tau_{j}$ have been labeled in preorder with the set $\pi_{j}$ where $\pi=\left\{\pi_{1}<\pi_{2}<\cdots<\pi_{k}\right\}$ is a 

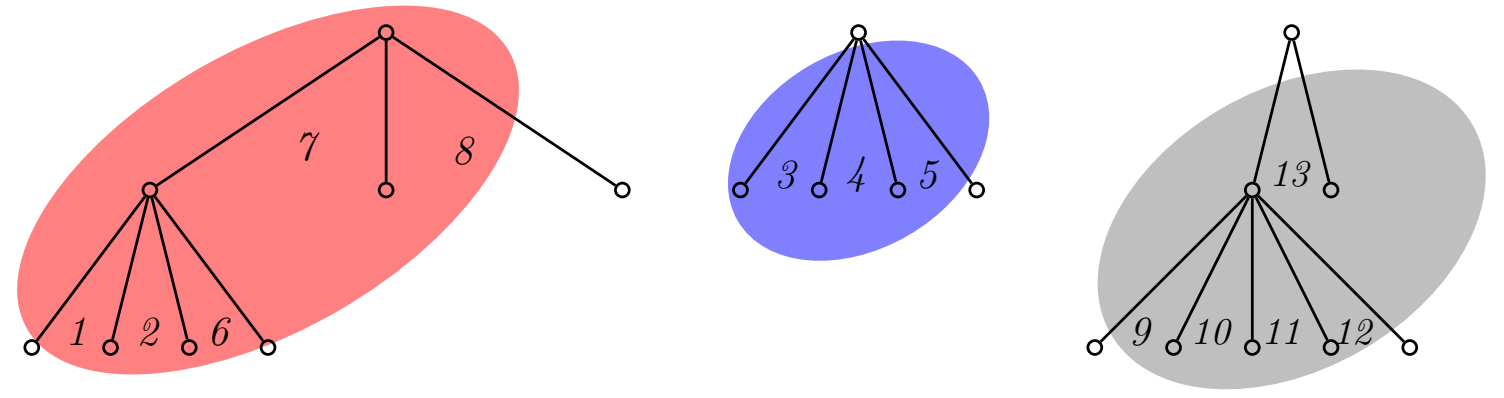

Figure 19. The recursive construction of the tree $T=\psi(\pi)$ for the noncrossing $(s-\mathbf{1})$ - partition $\pi=\{\{1,2,6,7,8\},\{3,4,5\},\{9,10,11,12,13\}\}$ with $s=(3,4,4,2,5)$.

noncrossing $(s-\mathbf{1})$-partition of $[n]$. Then there is a unique way to glue together the trees $\tau_{j}$ such that they form a planar rooted tree $T$ satisfying:

(1) No pair of trees $\tau_{i}$ and $\tau_{j}$ together form a larger left descendant subtree in $T$.

(2) The caverns of $T$ are labeled in preorder.

(3) $\operatorname{signat}(T)=s$.

Proof. By condition (1) we cannot glue two left descendant trees, say $\tau_{i}$ and $\tau_{j}$ with $i<j$, by attaching the root of $\tau_{i}$ to the left-most leaf of $\tau_{j}$ since they will form a larger left descendant tree. Now condition (2) and the fact of the partition $\pi$ is noncrossing implies that for $i<j$ either $\tau_{j}$ is nested in $\tau_{i}$ or all labels of $\tau_{j}$ are larger than all the labels of $\tau_{i}$. So given tree $T_{1}=\tau_{1}$ (whose $\min \pi_{1}=1$ ) there is a unique way of gluing $\tau_{2}$ to obtain $T_{2}$, its root needs to be glued in preorder to the leaf of $\tau_{1}$ immediately after the cavern labeled $\min \pi_{2}-1$ (if $\min \pi_{2}-1$ were not a cavern of $\tau_{1}$ then $\pi$ would not be a partition of $[n]$ ) we repeat this process inductively by gluing $\tau_{j}$ to $T_{j-1}$ to obtain $T_{j}$, its root glued in preorder to the leaf of $T_{j-1}$ immediately after the cavern labeled $\min \pi_{j}-1$. This construction preserves the preorder labeling and always glues $\tau_{j}$ to a leaf of $T_{j-1}$ that is not the leftmost leaf of another tree $\tau_{i}$. Now note that $\operatorname{signat}\left(\tau_{1}\right)=\tilde{s}_{1}$ and since $T_{1}=\tau_{1}$ is a left descendant tree then when attaching $\tau_{2}$ to form $T_{2}$ all the internal nodes of $T_{1}$ come in preorder before the internal nodes of $\tau_{2}$ hence $\operatorname{signat}\left(T_{2}\right)=\tilde{s}_{1} \oplus \tilde{s}_{2}$. In general when attaching $\tau_{j}$ to $T_{j-1}$ to obtain $T_{j}$, all the internal nodes of $\tau_{j}$ come last in preorder and hence $\operatorname{signat}\left(T_{j}\right)=\operatorname{signat}\left(T_{j-1}\right) \oplus \tilde{s}_{j}$, this inductively implies condition (3).

Given the conditions of Lemma 3.14 where the labels of the caverns in tree $\tau_{j}$ are the elements of $\pi_{j}$, we define $T:=\psi(\pi)$ where $T$ is the unique tree that the lemma predicts.

Theorem 3.15. Let $s \in \mathcal{C}$ omp be such that $s_{i} \geq 2$ for all $i$. The map $\phi: \mathcal{T}_{s} \rightarrow \mathcal{N C}_{s-1}$ is a bijection between $s$-trees and noncrossing $(s-1)$-partitions. The inverse of $\phi i s \psi$.

Proof. If $\pi=\phi(T)$ then by the definition of $\phi$ every block $\pi_{j} \in \pi$ gets its labels from the caverns of a maximal left descendant subtree $\tau_{j}$ in $T$. If $\tau_{j}$ has $\operatorname{signat}\left(\tau_{j}\right)=\tilde{s}_{j}$ then 


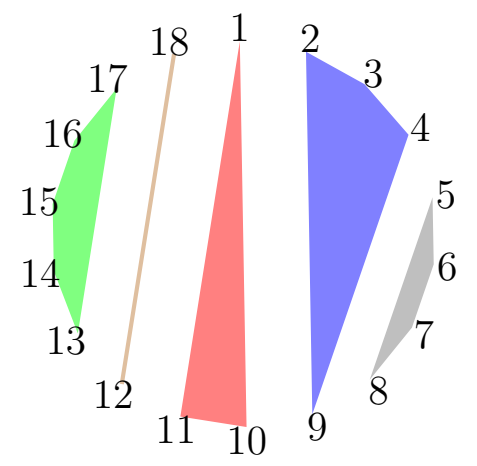

FigurE 20. Example of a complete $(3,4,4,2,5)$-matching

$s=\tilde{s}_{1} \oplus \tilde{s}_{2} \oplus \cdots \tilde{s}_{k}$ since all of these left descendant subtrees are consecutive in preorder by Lemma 3.12. But with these conditions together $\tilde{s}_{j}$ and $\pi_{j}$ recover $\tau_{j}$ uniquely and Lemma 3.14 says that there is a unique way to glue the $\tau_{j}$ together so $\psi(\pi)=T$. Similarly, if $T=\psi(\pi)$ then by construction a maximal left descendant subtree of $T$ have its caverns labeled by a block of $\pi$ and by Lemma 3.14 the labels of the caverns of $T$ are in preorder. Since each block of $\phi(T)$ is determined by the set of labels in the caverns of a maximal left descendant subtree when the caverns of $T$ have been labeled in preorder then $\phi(T)=\pi$.

3.5. Noncrossing matchings. A complete matching in the complete graph $K_{2 n}$ (the graph $(V, E)$ with vertex set $V=[2 n]$ and with edge set $E=\{S \subseteq[2 n]|| S \mid=2\})$ is a partition of the set $[2 n]$ in blocks of cardinality 2 . We say that the matching is noncrossing if this partition is noncrossing. It is known that the set of complete noncrossing matchings in $K_{2 n}$ is a Catalan family (see for example [35]). For any $n \geq 1$ the complete hypergraph $\mathcal{K}_{n}$ on $n$ vertices is the pair $(V, E)$ where $V=[n]$ is the set of vertices and $E=\{S \subseteq[2 n] \mid S \neq \emptyset\}$ the set of edges (Note that in this definition we consider vertices as edges of cardinality one).

Definition 3.16 (Complete noncrossing $s$-matchings). For $s \in \mathcal{C}$ omp, a complete noncrossing $s$-matching in $\mathcal{K}_{|s|}$ is a noncrossing partition $M$ of the set [|s|] such that $M$ satisfies $\left|M_{i}\right|=s(i)$ when we order the blocks of $M=\left\{M_{1}<M_{2}<\cdots<M_{a}\right\}$ in the minimal order (such that $\min M_{i}<\min M_{j}$ whenever $i<j$ ). We denote by $\mathcal{C M}_{s}$ the set of complete noncrossing $s$-matchings.

Figure 20 illustrates an example of a complete noncrossing $(3,4,4,2,5)$-matching.

Remark 3.17. Note that for $s \in \mathcal{C}$ omp both, $(s-\mathbf{1})$-partitions and complete $s$-matchings are partitions of different sets $([|s|-\ell(s)+1]$ and $[|s|]$ respectively). We use the name of complete $s$-matchings to denote the later type of partitions because they provide with a suitable generalization of the concept of complete matching in $K_{2 n}$. Also note that a given partition can be a $\nu$-paritition for different values of $\nu$ however a complete matching $M$ in $\mathcal{K}_{|s|}$ can only be a complete $s$-matching for the particular $s=\left(\left|M_{1}\right|,\left|M_{2}\right|, \ldots,\left|M_{a}\right|\right)$.

3.5.1. Bijection between s-trees and complete noncrossing s-matchings. For $T \in \mathcal{T}_{s}$ let $v_{0}, v_{1}, v_{2}, \ldots, v_{|s|}$ be the listing of the nodes of $T$ in preorder (where $v_{0}$ is the root of $T$ ) and 

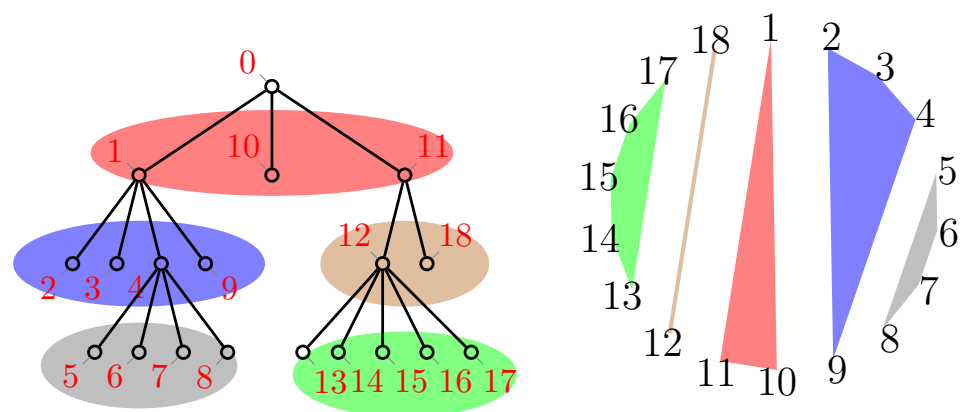

FiguRE 21. Example of the bijection between $s$-trees and noncrossing $s$ matchings. The nodes of the tree are ordered in preorder.

let $v_{i_{1}}, v_{i_{2}}, \ldots, v_{i_{a}}$ be the sublist of internal nodes in preorder. For each internal node $x$ let $\operatorname{child}(x)$ be the set of children of $x$. The matching $\varphi(T):=\left\{M_{1}, M_{2}, \cdots, M_{a}\right\}$ is defined as the partition with blocks $M_{k}=\left\{j \mid v_{j} \in \operatorname{child}\left(v_{i_{k}}\right)\right\}$. See Figure 21 for an example of the map $\phi$.

Lemma 3.18. Let $v_{i_{k}}$ be the $k$-th internal node of $T$ in preorder then

$$
\min M_{k}=i_{k}+1 \text {. }
$$

Consequently, $M_{1}, M_{2}, \ldots, M_{a}$ is the minimal order listing of $\phi(T)$.

Proof. An internal node and its leftmost child are consecutive in preorder, then $\min M(k)=$ $\min \left\{j \mid v_{j} \in \operatorname{child}\left(v_{i_{k}}\right)\right\}=i_{k}+1$.

Lemma 3.19. The partition $\varphi(T)$ is a complete noncrossing s-matching.

Proof. $\varphi(T)$ is noncrossing: Two different blocks of $\varphi(T)$ come from two different internal nodes $v_{i_{k}}$ and $v_{i_{l}}$, say with $v_{i_{k}}<v_{i_{l}}$ in preorder. If $v_{i_{k}}$ and $v_{i_{l}}$ are unrelated all the nodes in $\operatorname{child}\left(v_{i_{k}}\right)$ occur before the nodes in $\operatorname{child}\left(v_{i_{l}}\right)$ in preorder. If $v_{i_{k}}$ is an ancestor of $v_{i_{l}}$ then all the nodes in child $\left(v_{i_{l}}\right)$ are also descendant of exactly one child of $v_{i_{k}}$, hence they occur in between two preorder consecutive elements of child $\left(v_{i_{k}}\right)$ and $M_{l}$ is nested in $M_{k}$.

$\varphi(T)$ is a complete $s$-matching: By Lemma $3.18 M_{1}, M_{2} \ldots, M_{a}$ is the minimal order listing of $\varphi(T)$ and $\left|M_{k}\right|=\operatorname{deg}\left(v_{i_{k}}\right)=s(k)$ then

$$
\left(\left|M_{1}\right|,\left|M_{2}\right| \ldots,\left|M_{a}\right|\right)=(s(1), \ldots, s(a))=s .
$$

The inverse map $\gamma: \mathcal{C} \mathcal{M}_{s} \rightarrow \mathcal{T}_{s}$ is defined as follows. Let $M=\left\{M_{1}, M_{2}, \ldots, M_{a}\right\} \in \mathcal{C} \mathcal{M}_{s}$ be a complete noncrossing $s$-matching whose blocks are ordered according to the minimal order and recall that by definition $\left|M_{i}\right|=s(i)$. Define the tree $\gamma(M)$ as follows (see Figure 22 for an example):

(1) First, start with the root $r=v_{0}$ with $\left|M_{1}\right|$ children with increasing left-to-right labels $\left\{v_{j} \mid j \in M_{1}\right\}$ and call the resulting tree $\tau_{1}$.

(2) Attach an internal node to $\tau_{1}$ in $v_{\min M_{2}-1}$ with $s(2)$ children with increasing leftto-right labels $\left\{v_{j} \mid j \in M_{2}\right\}$ and call the resulting tree $\tau_{2}$. 
(3) In general, at the $j$ th step of the process we have a tree $\tau_{j-1}$ with $j-1$ internal nodes $v_{i_{1}}, \ldots, v_{i_{j}}$ ordered in preorder. The $j$ th tree $\tau_{j}$ is obtained from $\tau_{j-1}$ by attaching an internal node in $v_{\min } M_{j}-1$ with $s(j)$ children with increasing left-to-right labels $\left\{v_{j} \mid j \in M_{j}\right\}$.

(4) The process finishes after attaching the $a$ internal nodes $v_{i_{1}}, \ldots, v_{i_{a}}$.
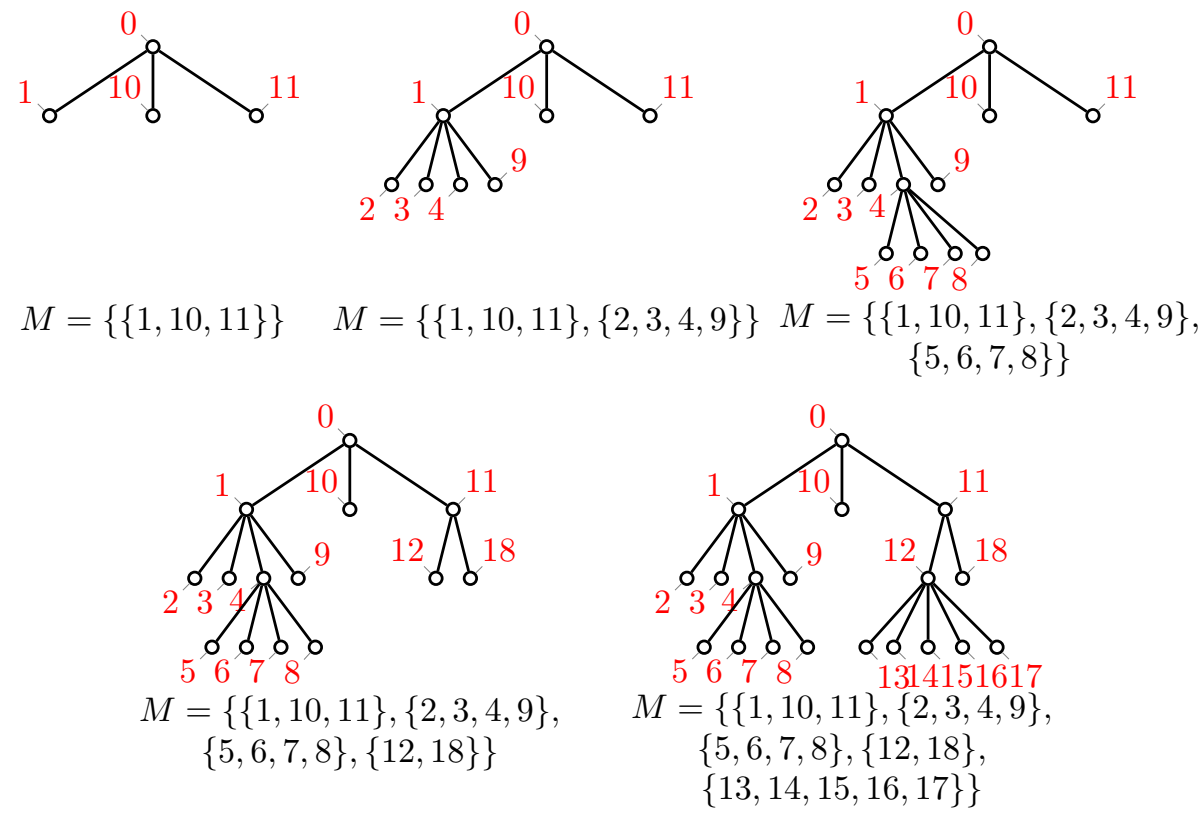

Figure 22. The recursive construction of the tree $\gamma(M)$ for the complete noncrossing $(3,4,4,2,5)$-matching $M=$ $\{\{1,10,11\},\{2,3,4,9\},\{5,6,7,8\},\{12,18\},\{13,14,15,16,17\}\}$.

We will use the following simple lemma.

Lemma 3.20. If $\left\{M_{1}, M_{2}, \ldots M_{k}\right\}$ is a noncrossing partition whose blocks are ordered according to the minimal order, then $M_{k}$ and $\bigcup_{i=1}^{k-1} M_{i}$ are also noncrossing.

Lemma 3.21. $\gamma(M)$ is an s-tree with all its nodes labeled in preorder.

Proof. To see that the map is well-defined note that in the step $j$ the node $v_{\min M_{j}-1}$ has already been attached to the tree $\tau_{j-1}$ otherwise there is a block $M_{k}$ with $\min M_{j}-1 \in M_{k}$ and $k>j$ implying min $M_{k}<\min M_{i}$ contradicting the minimal ordering of $M$.

In the step $j$ we are attaching leaves with the labels in $M_{j}$. Since $M$ is noncrossing by Lemma $3.20 M_{j}$ and $\bigcup_{i=1}^{j-1} M_{i}$ are noncrossing implying that the labels of $M_{j}$ are either nested or are all greater than the labels in $\bigcup_{i=1}^{j-1} M_{i}$. This implies that when we attach the vertices $\left\{v_{i} \mid i \in M_{j}\right\}$ as children of $v_{\min M_{j}-1}$ in $\tau_{j}$, the labels of the nodes in $\tau_{j}$ are still consistent with preorder.

Theorem 3.22. The map $\varphi: \mathcal{T}_{s} \rightarrow \mathcal{C M}_{s}$ is a bijection between s-trees and complete noncrossing s-matchings. The inverse of $\varphi$ is $\gamma$. 
Proof. After labeling the nodes of $T$ in preorder. Lemma 3.18 and the constructive definition of $\gamma$ imply that the labels of the internal nodes in $T$ and $\gamma(\varphi(T))$ are the same. Also, the constructive definition of both $\varphi$ and $\gamma$ imply that for each internal node $v_{i_{k}}$ the labeled set of children in $T$ and $\gamma(\varphi(T))$ are equal. These two conditions determine $T$ completely, therefore $T=\gamma(\varphi(T))$.

Similarly, in the construction of the tree $\gamma(M)$ the labels of a block $M_{k}$ are the children of an internal node. Since every block of $\varphi(T)$ is the set of labels of the children of an internal node then we have that $\varphi(\gamma(M))=M$.

\section{The Fundamental RECURREnCE}

Trees have an inherent recursive nature, in the case of $s$-trees the recursion is very natural: Any $s$-tree $T$ can be constructed as $T=\left[T_{1}, T_{2}, \ldots, T_{s(1)}\right]$ for $s(1)$ suitable trees $T_{1}, T_{2}, \ldots, T_{s(1)}$ satisfying

$$
\operatorname{signat}(T)=(s(1)) \oplus \operatorname{signat}\left(T_{1}\right) \oplus \cdots \oplus \operatorname{signat}\left(T_{s(1)}\right) .
$$

From this we can obtain a recursion for the number $C_{s}$ of $s$-Catalan structures:

$$
C_{s}=\sum_{(s)} C_{s_{1}} C_{s_{2}} \cdots C_{s_{s(1)}}
$$

where the sum is over all sequences $\left(s_{1}, s_{2}, \ldots, s_{s(1)}\right)$ of compositions such that $s=(s(1)) \oplus$ $s_{1} \oplus s_{2} \oplus \cdots \oplus s_{s(1)}$ and where $C_{\emptyset}=1$.

Recursion (4.1) is at the heart of $s$-Catalan combinatorics and it is a generalization of the classical recursion for the number $C_{n}$ of Catalan objects. By letting $C_{n}:=C_{\left(2^{n}\right)}$ and $C_{0}=1$ in (4.1) we obtain the classical recursion:

$$
C_{n+1}=\sum_{k=0}^{n} C_{k} C_{n-k} .
$$

4.1. Catalan decompositions. For a family $\mathcal{A}_{s}$ of $s$-Catalan objects recursion (4.1) can be realized by obtaining a rule of decomposition of an $s$-object $A=\left[A_{1}, A_{2}, \ldots, A_{s(1)}\right]$ into $s(1)$ objects $A_{1} \in \mathcal{A}_{s_{1}}, A \in \mathcal{A}_{s_{2}}, \ldots, A_{s(1)} \in \mathcal{A}_{s_{s(1)}}$ such that $s=(s(1)) \oplus s_{1} \oplus \cdots \oplus s_{s(1)}$. We call such a rule a Catalan decomposition.

Whenever we have a pair of families of objects $\mathcal{A}_{n}$ and $\mathcal{B}_{n}$ with $\mathcal{A}_{\emptyset}=\left\{A_{\emptyset}\right\}$ and $\mathcal{B}_{\emptyset}=\left\{B_{\emptyset}\right\}$ both with known Catalan decomposition rules. Then a family of bijections $\xi_{s}: \mathcal{A}_{s} \rightarrow \mathcal{B}_{s}$ are easily defined recursively by:

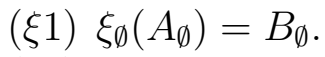

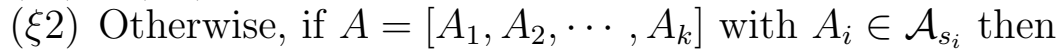

$$
\xi_{s}(A):=\left[\xi_{s_{1}}\left(A_{1}\right), \xi_{s_{2}}\left(A_{2}\right), \ldots, \xi_{s_{k}}\left(A_{k}\right)\right]
$$

Such family of bijections sometimes can be described in a nicer and direct way without using recursion. We illustrate in Section 4.3 and Section 4.4 the use of the recursion (4.1) with the examples of angulations of a convex polygon and parenthesizations of a word. 


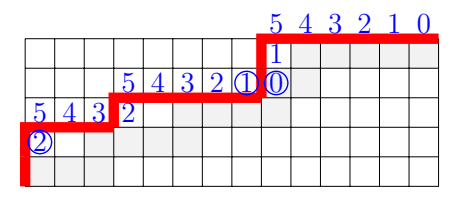

$$
s=(3,4,4,2,5)
$$

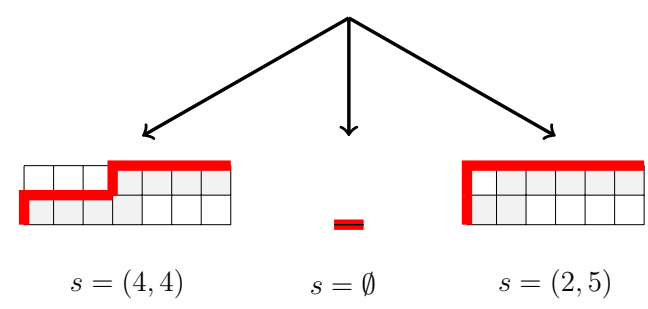

Figure 23. Example of the Catalan decomposition of an s-Dyck path

On the other hand if there is a family of bijections $\xi_{s}: \mathcal{A}_{s} \rightarrow \mathcal{B}_{s}$ where the Catalan decomposition rule for the objects in $\mathcal{A}_{s}$ is known, then we might be able to transport this rule to the objects in $\mathcal{B}_{s}$ using the maps $\xi_{s}$. This idea can be used to describe Catalan decompositions for other families of $s$-Catalan objects, like $s$-Dyck paths, 312-avoiding Stirling $(s-1)$-permutations, noncrossing $s$-partitions, and complete noncrossing $s$-matchings.

4.2. Catalan decomposition for Dyck paths. The Catalan decomposition of an $s$ Dyck path $D$ is relatively simple. We remove the first north step, which corresponds to the root of the corresponding tree, and label the lattice points of the resulting path $\bar{D}$ (except the last one) by their horizontal distance to the Ribbon shape determined by $s$. That is, a lattice point $p$ is labeled by the maximal number of east steps that can be added to $p$ without crossing the Ribbon shape. ${ }^{1}$ For the example in Figure 23 we get the sequence

$$
2,5,4,3,2,5,4,3,2,1,0,1,5,4,3,2,1,0 .
$$

We cut the path $\bar{D}$ at the first appearances of $s(1)-1, s(1)-2, \ldots, 1,0$ (marked in red in the sequence and highlighted in Figure 23). The resulting sequence $\left(D_{1}, D_{2}, \cdots, D_{s(1)}\right)$ of Dyck paths (possibly equal to the identity Dyck path $E$ ) form the Catalan decomposition of $D$, where the signature of $D_{i}$ is defined as the sub-sequence of $s$ determined by the rows of the north steps in $D_{i}$. Their composition is defined as

$$
\left[D_{1}, D_{2}, \ldots, D_{k}\right]:=N D_{1} D_{2} \cdots D_{k} .
$$

The validity of this decomposition follows from the correspondence between $s$-trees and $s$-Dyck paths, and the area labeling in Section 3.2.1.

\footnotetext{
${ }^{1}$ We are adding an extra "imaginary" box on top of the top-right box of the Ribbon shape to make this definition work for points on top of the grid.
} 


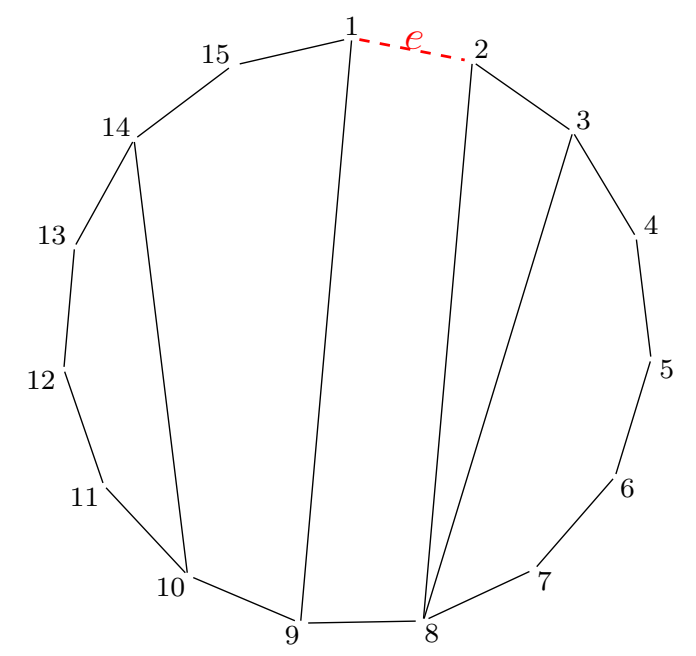

Figure 24. An angulation of $P(15)$

4.3. Angulations of a polygon. For $n \in \mathbb{N}$ we denote by $P(n+2)$ a convex polygon with $n+2$ vertices. A diagonal of $P(n+2)$ is a straight line joining any two nonadjacent vertices of the polygon. An angulation of $P(n+2)$ is a partition of the interior of $P(n+2)$ into smaller convex polygons using a set of noncrossing diagonals. See Figure 24 for an example of an angulation of $P(15)$ into 5 parts.

Label all the vertices of $P(n+2)$ clockwise let $e$ be the edge between the vertices 1 and 2 and let $A$ be an angulation of $P(n+2)$. It is enough to remove $e$ to reveal the Catalan decomposition of $A$ into angulations of smaller polygons. An edge is considered as the polygon $P(2)$ with a unique triangulation. Then the set $\mathcal{A P}$ of angulations of polygons has the Catalan recursive structure. It is then a simple task to define the signature of an angulation $A$. Indeed, if removing $e$ gives angulations $A_{1}, A_{2}, \ldots, A_{k}$ traveling counterclockwise then

$$
\operatorname{signat}(A):=(k) \oplus \operatorname{signat}\left(A_{1}\right) \oplus \operatorname{signat}\left(A_{2}\right) \oplus \cdots \oplus \operatorname{signat}\left(A_{k}\right),
$$

where the signature of a single edge is $\operatorname{signat}(\mathbf{m})=\emptyset$. We denote by $\mathcal{A} \mathcal{P}_{s}$ the set of $s$-angulations of $P(|s|-\ell(s)+2)$, that is the set of angulations $A$ with $\operatorname{signat}(A)=s$.

Using this recursive construction we can easily find a bijection between the $s$-trees and $s$-angulations of $P(|s|-\ell(s)+2)$ using the strategy described above. We leave the proof to the interested reader, but illustrate in Figure 25 an example of this bijection when $s=(3,4,4,2,5)$ and the tree $T$ is the one in our example of Figure 6 .

4.4. Parenthesizations. Starting with a word $w$ with $b$ letters consider the word $w^{\prime}$ of length $2 a+b$ obtained by inserting $a$ left parenthesis and $a$ right parenthesis. For example let $a=3$ and $b=5$, so for the word $w=* * * * *$ we can insert left and right parenthesis in any way, say $\left.w_{1}^{\prime}=\right) *\left((*) *(* *)\right.$ or $w_{2}^{\prime}=(*((* *) *) *)$. A (proper) parenthesization of a word $w$ with $b$ letters is the insertion of the same number $a$ of left and right parenthesis with the 


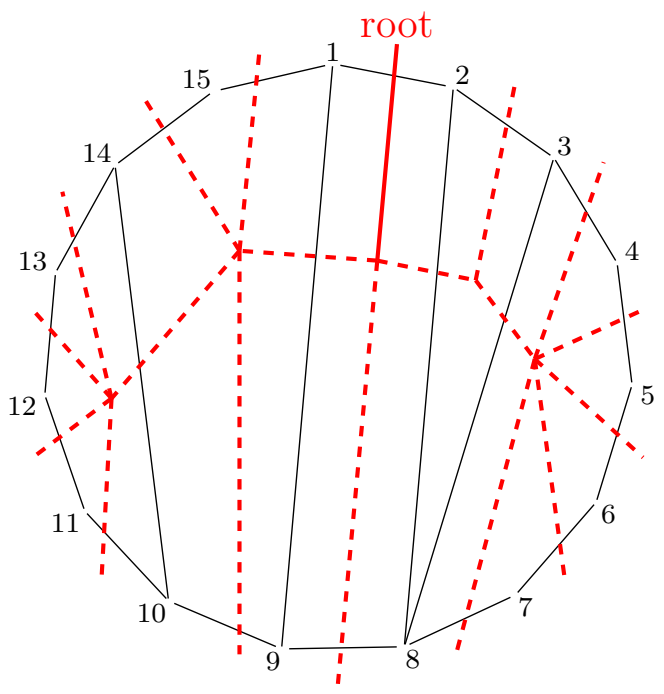

Figure 25. Example of the bijection between $s$-trees and $s$-angulations

conditions that for any $i \in[2 a+b]$ the prefix $w(1) w(2) \cdots w(i)$ contains at least as many left parenthesis as right parenthesis and that there cannot be an $i$ such that $w(i) w(i+1)=()$.

The word $w_{2}^{\prime}$ above is an example of a good parenthesization, another example is the word

$$
w=(* *(* * * *) *) *((* * * * *) *) .
$$

A segment $u$ of a word $w=w(1) w(2) \cdots w(k)$ is a subword of $w$ of the form $u=$ $w(i+1) w(i+2) \cdots w(i+\ell)$, i.e., all the letters of $u$ are adjacent in $w$. A block in a properly parenthesized word $w$ is a properly parenthesized segment $u$ that is inclusion maximal with respect to the property that any proper prefix in $u$ of the form $u(1) u(2) \cdots u(r)$ with $r<\ell$ contains strictly more left parenthesis than right parenthesis. For the example (4.3) there are three blocks, namely $(* *(* * * *) *), *$ and $((* * * * *) *)$.

It is easy to see out of the definition above that every properly parenthesized word factors into blocks in the form $w=B_{1} B_{2} \cdots B_{k}$ (see example (4.3)) and that there is a unique parenthesization of the word $*$ with $b=1$ and $a=0$. So parenthesizations satisfy the recursion (4.1) and are then Catalan objects. Define signat $(*)=\emptyset$ and for the parenthesized word $w=B_{1} B_{2} \cdots B_{k}$ define its signature $\operatorname{signat}(w)$ recursively as

$$
\operatorname{signat}(w)=(k) \oplus \operatorname{signat}\left(B_{1}\right) \oplus \cdots \oplus \operatorname{signat}\left(B_{k}\right) .
$$

For $w$ of example (4.3) we have that $\operatorname{signat}(w)=(3,4,4,2,5)$. For $s \in \mathcal{C}$ omp, an $s$ parenthesization is a properly parenthesized word $w \operatorname{such}$ that signat $(w)=s$. We denote $\mathcal{P} \mathcal{T}_{s}$ the set of $s$-Parenthesizations of a word of length $|s|-\ell(s)+1$.

As in the case of $s$-angulations, $s$-trees can be recursively mapped bijectively to $s$ parenthesizations using their recursive constructions. Again, we leave the proof to the interested reader and illustrate the bijection in Figure 26 when $s=(3,4,4,2,5)$ and the 


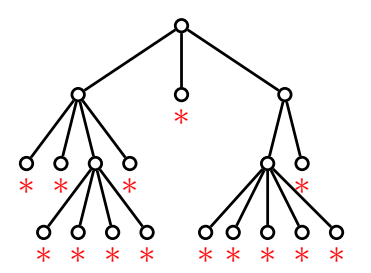

$$
((* *(* * * *) * \stackrel{\circ}{*} *((* * * * *) *))
$$

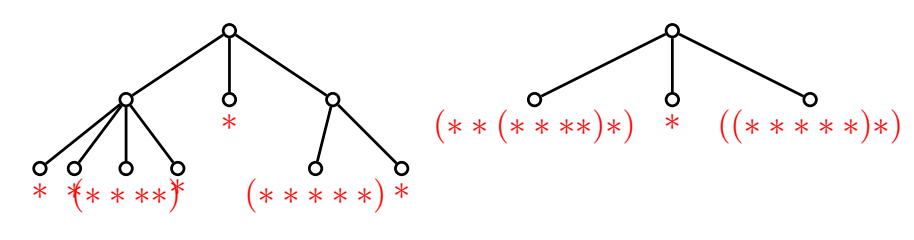

$(* *(* * * *) *) *((* * * * *) *)$

without outermost parenthesis

FiguRE 26. Example of the bijection between $s$-trees and $s$-parenthesizations

tree $T$ is the one in our running example of Figure 6. Note from the Figure that at the end of the process we always omit the outermost parenthesis since it is redundant.

\section{ENUMERATION}

Let $\lambda=\left(\lambda_{1} \geq \lambda_{2} \geq \cdots \geq \lambda_{\ell}\right)$ be a partition. We say that a partition $\lambda^{\prime}=\left(\lambda_{1}^{\prime} \geq \lambda_{2}^{\prime} \geq\right.$ $\cdots \geq \lambda_{\ell}^{\prime}$ ) fits $\lambda$ if the tableau of $\lambda^{\prime}$ is contained in the tableau of $\lambda$, in other words, if $\lambda_{i}^{\prime} \leq \lambda_{i}$ for all $i$.

Theorem 5.1 (Kreweras [16]). The number $P(\lambda)$ of partitions fitting a partition $\lambda$ is counted by the determinant formula

$$
P(\lambda)=\operatorname{det}\left(\begin{array}{c}
\lambda_{j}+1 \\
j-i+1
\end{array}\right)
$$

In particular, for stair case partitions the numbers $P(\lambda)$ recover the classical Catalan numbers.

5.1. $s$-Catalan numbers. Note that every composition $s=\left(s_{1}, \ldots, s_{a}\right)$ defines a partition $\lambda^{s}=\left(\lambda_{1}^{s} \geq \cdots \geq \lambda_{a-1}^{s}\right)$ such that $\lambda_{j}^{s}=\sum_{i=1}^{a-j}\left(s_{i}-1\right)$, that is basically the shape of the upper shape that is left when in a $(|s|-1) \times a$ grid we remove the central ribbon determined by $s$. In the example of Figure 8 we have that $\lambda^{(3,4,4,2,5)}=(9,8,5,2)$. It is not hard to see that in the same manner as the ribbon shape determined by $s$ describes a partition $\lambda^{s}$, every $s$-Dyck path describes a partition $\lambda^{\prime} \leq \lambda^{s}$. Theorem 5.1 gives then a determinantal formula for the number of $s$-Dyck paths.

Corollary 5.2 (Kreweras). The s-Catalan number is counted by the determinant formula

$$
\left|\mathcal{C}_{s}\right|=\operatorname{det}\left(\begin{array}{c}
\sum_{i=1}^{a-j}\left(s_{i}-1\right)+1 \\
j-i+1
\end{array}\right) \text {. }
$$

5.2. s-Narayana numbers. The Catalan number $C_{n}=\frac{1}{n+1}\left(\begin{array}{c}2 n \\ n\end{array}\right)$ can be refined by the Narayana numbers $N(n, k)$. These numbers are indexed by an extra parameter $1 \leq k \leq n$, 
can be explicitly computed by the simple formula

$$
N(n, k)=\frac{1}{n}\left(\begin{array}{l}
n \\
k
\end{array}\right)\left(\begin{array}{c}
n \\
k-1
\end{array}\right)
$$

and satisfy

$$
C_{n}=N(n, 1)+N(n, 2)+\cdots+N(n, n) .
$$

The Narayana numbers count Catalan objects that satisfy certain property which depends on the family that they belong to. For instance, $N(n, k)$ is the number of (see [29, Chapter 2] for further information):

(1) planar binary trees with $n$ internal nodes and $k$ most left leaves;

(2) Dyck paths in an $n \times n$ grid with $k$ peaks;

(3) 312-avoiding permutations of $[n]$ with $k-1$ ascents;

(4) noncrossing partitions of $[n]$ with $k$ blocks;

(5) noncrossing matchings of $[2 n]$ containing $k$ matched pairs of the form $(i, i+1)$.

All these combinatorial interpretations can be naturally extended to the generalized $s$ Catalan families. The $s$-Narayana number is defined as the number of elements in the following families. We remark that they correspond to each other under the bijections presented in Section 3 and Appendix A.

(1) $s$-trees with $k$ most left leaves;

(2) $s$-Dyck paths with $k$ peaks;

(3) 312-avoiding Stirling $(s-\mathbf{1})$-permutations with $k-1$ ascents;

(4) noncrossing $(s-\mathbf{1})$-partitions with $k$ blocks;

(5) Complete noncrossing $s$-matchings with $k$ parts satisfying $\min M_{i}+1 \in M_{i}$.

As far as we know there are no simple formulas to count these combinatorial objects in this general setting. However, refinements of the $s$-Narayana numbers have already been studied in the literature, for instance in the case of Fuss-Narayana numbers and rational Narayana numbers [3, 18, 19].

\section{Signature Generalizations of PERMUtATions AND PARKIng FUnCTiOns}

6.1. $s$-generalized permutations. As mentioned in the introduction, permutations of $[n]$ are in bijection with increasingly labeled planar binary trees with $n$ internal nodes. A natural generalization of permutations in terms of signatures is the collection of permutations corresponding to $(s+\mathbf{1})$-increasing trees. As explained in Section 3.3.1, such permutations are in correspondence with Stirling $s$-permutations, which were already considered and studied by Gessel and Stanley back in the seventies [10] for the special case $s=(2, \ldots, 2)$. As described above, these are multipermutations of the multiset $\left\{1^{s(1)}, 2^{s(s(2))}, \ldots, a^{s(a)}\right\}$ that avoid the pattern 212 ; see $[17,13,27,26,25,11,6,32]$ for further studies on such permutations.

The cardinality of the set $\mathcal{S P}_{s}$ of Stirling $s$-permutations generalizes the factorial numbers. To be more precise, from a permutation in $\mathcal{S P} \mathcal{P}_{(s(1), s(2), \ldots, s(k-1))}$ we can obtain a permutation in $\mathcal{S P}_{s \oplus(s(k))}$ by inserting the $s(k)$ consecutive occurrence of the label $k k \cdots k$ 
in any of the $s(1)+s(2)+\cdots+s(k-1)+1$ possible positions. Inductively and starting in $\mathcal{S P} \mathcal{P}_{(s(1))}=\{11 \cdots 1\}$ this implies that

$$
\left|\mathcal{S P}_{s}\right|=1 \cdot(s(1)+1) \cdot(s(1)+s(2)+1) \cdots(s(1)+s(2)+\cdots+s(a-1)+1)=:|s| !^{s},
$$

and we read $|s| !^{s}$ as $|s| s$-factorial. Note that if $s=\left(1^{n}\right)$ then $|s| !^{s}=n !$ and if $s=\left(2^{n}\right)$ then $|s| !^{s}=1 \cdot 3 \cdots(2 n-1)=(2 n-1)$ !!, the factorial and double-factorial numbers.

In forthcoming work [5], the first author and Viviane Pons study a generalization of the weak order on permutations for the set of Stirling $s$-permutations. They call this generalized order the $s$-weak order and show that it has the structure of a lattice. They also investigate geometric realizations of the $s$-weak order in terms of polytopal subdivisions of the classical permutahedron.

6.2. $s$-generalized parking functions. A parking function of $n$ is a sequence of nonnegative integers $\left(p_{1}, p_{2}, \ldots, p_{n}\right)$ with the property that after being rearranged in weakly increasing order $p_{j_{1}} \leq p_{j_{2}} \leq \cdots \leq p_{j_{n}}$ they satisfy $p_{j_{i}}<i$ for all $i \in[n]$. We denote by $\mathcal{P} \mathcal{F}_{n}$ the set of parking functions of $n$. Parking functions were studied by Konheim and Weiss [14] were they used the following equivalent description to define them:

Let $C_{1}, \ldots, C_{n}$ be a collection of cars to be parked in order, car $C_{i}$ before car $C_{i+1}$, in a one-way street in a consecutive sequence of parking spots marked $P_{0}, \ldots, P_{n-1}$. The driver of car $C_{i}$ has a preferred spot $p_{i}$ where he would like to park. In step $i$ the driver of car $C_{i}$ drives up to the spot $p_{i}$, parking there if the spot is available, or otherwise continuing until the next available spot. If the spot $p_{i}$ and all the following parking spots are occupied then $C_{i}$ is unable to park. A parking function is a sequence $\left(p_{1}, p_{2}, \ldots, p_{n}\right)$ of parking preferences where all the cars are able to park.

It is not difficult to verify that the two definitions of parking function stated above are equivalent. There is another way to construct parking functions. We can obtain parking functions by decorating Dyck paths with the elements of $[n]$.

A decorated Dyck path is a Dyck path in which the north steps have been decorated from left to right with a permutation of $[n]$ in such a way that consecutive north steps have increasing labels (see Figure 27). Let $\widehat{\mathcal{D P}}_{n}$ denote the set of decorated Dyck paths of $[n]$. In Figure 1 the reader can see the example of $\widehat{\mathcal{D P}}_{3}$.

It turns out that the sets $\mathcal{P} \mathcal{F}_{n}$ and $\widehat{\mathcal{D P}}_{n}$ are in bijection and that decorated Dyck paths are a convenient way to encode parking functions. The bijection we will describe is common in the literature, see for example [8]. The function $P: \widehat{\mathcal{D P}}_{n} \rightarrow \mathcal{P} \mathcal{F}_{n}$ that provides a parking function $P(D)$ starting with a decorated Dyck path $D$ is defined as follows: the value of $p_{i}$ is the number of east steps that are in $D$ to the left of the north step decorated with the letter $i$. The function $P$ is bijective and its inverse is also easy to describe. In the example of Figure 27 we have for example that $p_{1}=0$ because there are no east steps before the north step labeled 1 and $p_{4}=5$ since there are 5 east step before the north step labeled 4.

Perhaps the easiest way to generalize the concept of parking function is using the concept of a decorated Dyck path. For a composition $s$ let $\widehat{\mathcal{D P}}$ be the set of $s$-Dyck paths whose north steps have been decorated with a permutation of $[a]$ in such a way that consecutive 


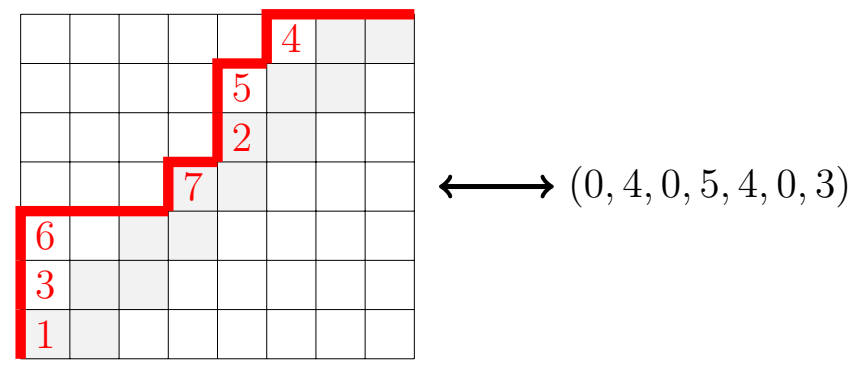

FiguRE 27. Example of a decorated Dyck path and its corresponding parking function

north steps have increasing labels. The discussion above illustrates that the set $\widehat{\mathcal{D P}}_{s}$ is also in bijection with a more general set of parking functions. For a weak composition $\mu$ a $\mu$-parking function is a sequence of nonnegative integers $\left(p_{1}, p_{2}, \cdots, p_{a}\right)$ such that after being rearranged in weakly increasing order $p_{j_{1}} \leq p_{j_{2}} \leq \cdots \leq p_{j_{a}}$ they satisfy $p_{j_{1}}=0$ and

$$
p_{j_{i}} \leq \sum_{i=1}^{i-1} \mu(i) \text { for all } i>1 \text {. }
$$

We denote by $\mathcal{P} \mathcal{F}_{\mu}$ the set of $\mu$-parking functions. By the same reasoning as above we have that there is a bijection $\widehat{\mathcal{D P}}_{s} \rightarrow \mathcal{P F}_{s-\mathbf{1}}$. $\mu$-parking functions have already appear in the literature with the name of generalized parking functions, vector parking functions or $\mu$-parking functions. They were originally studied by Pitman and Stanley [36] and Yan $[39,38]$ in a slightly more general form. This more general form can be obtained within this framework if we allow 0 values in our signatures and what we call a $\mu$-parking function is what appears as a (1) $\oplus \mu$-vector parking function in the literature. The case $s-\mathbf{1}=\left(\mathbf{1}^{\mathbf{n}}\right)$ returns the classical concept of parking functions counted by $(n+1)^{n-1}$ and in general if $s$ is a rational signature for $(a, b)$ relatively prime then Armstrong, Loehr and Warrington [2] show that $\left|\mathcal{P} \mathcal{F}_{a, b}\right|=b^{a-1}$, this includes the case $a=n$ and $b=k n+1$ or $s-\mathbf{1}=\left(\mathbf{k}^{\mathbf{n}}\right)$ of Fuss-Catalan parking functions. The rational signatures are basically the cases were a nice product formula is known and to find formulas for general signatures seems to be a difficult task. In [9] Gaydarov and Hopkins generalize the work in [28] to provide a bijection between vector parking functions and a set of trees that they call rooted plane trees.

We finish this section with the remark that even thought in the literature it is most common to represent parking functions as decorated Dyck paths, we argue that is more natural and probably more convenient from the recursive point of view to use the perspective of $s$-trees to represent a parking function. A decorated $s$-tree is an $s$-tree such that all the internal nodes are decorated with distinct elements of $[a]$ in such a way that an internal node that is the left-most child of its parent has a larger label. Let $\widehat{\mathcal{T}}_{s}$ be the set of decorated $s$-trees, then is clear from Theorem 3.15 that we have a bijection $\widehat{\mathcal{T}}_{s} \cong \widehat{\mathcal{D P}}_{s} \cong \mathcal{P} \mathcal{F}_{s-\mathbf{1}}$. In Figure 28 we illustrate an example of a decorated $s$-tree corresponding to the $\left(1^{7}\right)$-parking 


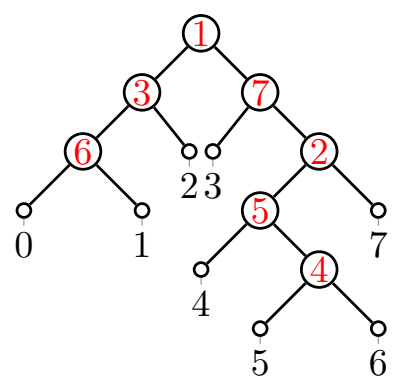

FiguRE 28. Example of the decorated $s$-tree corresponding to the parking function of Figure 27

function $(0,4,0,5,4,0,3)$. Note that the associated parking function can be obtained by labeling the leaves left-to-right from 0 to $|s-\mathbf{1}|+1$, then $p_{i}$ is equal to the label of the leaf that is the leftmost descendant of the internal node decorated $i$.

\section{Relation to the rational constructions of Armstrong, Rhoades and WILLIAMS}

The constructions in this article generalize the rational Dyck paths of Armstrong, Rhodes and Williams [3] to more general signatures $s$. A natural question that arises is whether our constructions for noncrossing $s$-partitions, complete noncrossing $s$-matchings and $s$ angulations of a $(|s|-\ell(s)+2)$-gon specialize to their rational constructions for the same type of objects in [3] when $s$ is a rational signature. The answer to this question happens to be on the negative side. Even though in some examples the two constructions coincide they are in general different. In this section we discuss the difference between the constructions and in particular we illustrate the situation in the case of rational noncrossing partitions.

Let $(a, b)$ be a pair of relatively prime positive numbers and $s$ the corresponding rational signature defined as in equation 3.1. In [3], the authors define a family of inhomogeneous $(a, b)$-noncrossing partitions (that we will denote here by $\widetilde{\mathcal{N C}}_{(a, b)}$ ), a family of homogeneous $(a, b)$-noncrossing partitions (denoted here by $\left.\widetilde{\mathcal{C M}}_{(a, b)}\right)$ and a family of rational dissections of a polygon (denoted here by $\widetilde{\mathcal{A P}}_{(a, b)}$ ). The constructions of $\widetilde{\mathcal{N C}}_{(a, b)}, \widetilde{\mathcal{C M}}_{(a, b)}$ and $\widetilde{\mathcal{A P}}_{(a, b)}$ rely on a "laser" construction. A laser through the point $\left(x_{0}, y_{0}\right)$ is the half-ray in the grid $b \times a$ with starting point $\left(x_{0}, y_{0}\right)$ and slope $\frac{a}{b}$, i.e., a half-ray given by the equation $y=y_{0}+\frac{a}{b}\left(x-x_{0}\right)$ with $x \geq x_{0}$. See the Figure 29 for examples of lasers that go through the points $(0,0),(2,2),(6,3)$ and $(10,4)$ with slope $\frac{5}{13}$.

We now describe the construction of $\widetilde{\mathcal{N C}}_{(a, b)}$. For a given $(a, b)$-Dyck path $D$ we label the right ends of the east steps of $D$ from left to right with the labels $1,2, \ldots,(b-1)$. For every consecutive sequence of north steps in $D$ fire a laser through the lower point where the sequence starts. The lasers give a topological decomposition of the reqion between $D$ and the diagonal $y=\frac{a}{b} x$ and then we can define a partition $\pi(D)$ by letting $i$ and $j$ to be in the same block if they belong to the same connected component. In [3, Proposition $6.1]$ it is shown that $\pi(D)$ is a noncrossing partition of $[b-1]$ and that $\pi$ is an injective 
map, which allow them to define rational noncrossing partitions. In Figure 29 we illustrate an example of this construction starting with the $(5,13)$-Dyck path (or $(3,4,3,4,3$-Dyck path in our language) $D=N^{2} E^{2} N E^{4} N E^{4} N E^{3}$. The noncrossing partition obtained from the construction in [3] is $\pi(D)=\{\{1,2,5,6,9,10\},\{3,4\},\{7,8\},\{11,12\}\}$ while the noncrossing partition obtained from $D$ using our constructions by first identifying the $(3,4,3,4,3)$-tree $\zeta(D)$ associated to $D$ and then finding the noncrossing $(3,4,3,4,3)$ partition $\phi(\zeta(D))=\{\{1,2,5,6,10\},\{3,4\},\{7,8,9\},\{11,12\}\}$, showing that our constructions arrive to different objects. Note that not only the noncrossing partitions associated to $D$ are different but also the sets $\widetilde{\mathcal{N C}}_{(5,13)}$ and $\mathcal{N C}_{(2,3,2,3,2)}$ of rational noncrossing partitions are different. The partition $\pi(D)=\{\{1,2,5,6,9,20\},\{3,4\},\{7,8\},\{11,12\}\}$ has parts of sizes $\mu(\pi(D))=(6,2,2,2)$, so in particular, it is not refined by $s-\mathbf{1}=(2,3,2,3,2)$ and

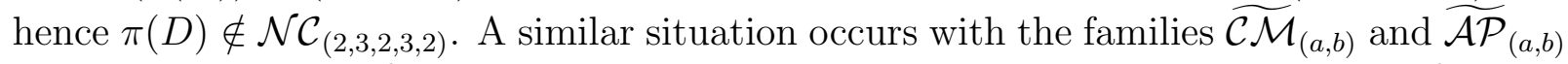
since they are also defined by the laser construction and our constructions have definitions that reflect the underlying tree structure of the objects instead.

In [3] Armstrong, Rhodes and Williams pose as an open problem to give characterizations of the families $\widetilde{\mathcal{N C}}_{(a, b)}, \widetilde{\mathcal{C M}}_{(a, b)}$ and $\widetilde{\mathcal{A P}}_{(a, b)}$ that are somewhat more natural and do not rely in a laser construction. One advantage of our constructions is precisely that they are independent of the laser construction and that they happen to reflect the natural tree structure of $s$-Catalan families. One advantage in the laser constructions however is that they happen to have certain rotational symmetry. Indeed, it was proven in [3, Proposition $5.2]$ that $\widetilde{\mathcal{C M}}_{(a, b)}$ is closed under rotation and the same property is conjecturally true for $\widetilde{\mathcal{N C}}_{(a, b)}$. It is not difficult to check that the families $\mathcal{C} \mathcal{M}_{s}$ and $\mathcal{N C}_{s-1}$ are in general not closed under rotation unless $s=\left(k^{a}\right)$ for some value of $k$, since the rotation operation also have the effect of permuting the entries of the signature $s$. Hence if we want to have rotational symmetry we need to go to a larger family of objects that includes all the possible permutations of the signature. Finally, a clear advantage of our constructions is that we produce, as a byproduct, a family of $(a, b)$-trees that is central in the new rational picture and that it was not defined before in the literature. We claim that this central object could play an important role when studying families of rational Catalan objects for its inherent recursive nature. For example the $(a, b)$-trees also allow us to obtain the notion of Stirling $(a, b)$-permutations that were not considered before inside this picture.

\section{Algebraic structures on signature objects}

In a forthcoming article we study various algebraic structures that are related to the set $\mathcal{T}=\bigcup_{s \in \mathcal{C} o m p} \mathcal{T}_{s}$ of planar rooted trees and such that after applying the corresponding algebraic operations some properties of signatures are preserved.

The structure that is most notably related to $\mathcal{T}$ is the one that models the category of nonsymmetric operads, see [21] for the context and notation. Let $\mathbb{N}$-Mod be the category of $\mathbb{N}$-graded (or commonly known as arity graded) vector spaces and graded preserving linear maps over some field $\mathbf{k}$. Over the monoidal category of endofunctors $F: \mathbb{N}$-Mod $\rightarrow \mathbb{N}$-Mod with composition of endofunctors and whose identity $\mathbb{I}$ is the identity endofunctor we have a monad $\mathcal{T}: \mathbb{N}$-Mod $\rightarrow \mathbb{N}$-Mod (we are abusing notation using $\mathcal{T}$ here also but hope 

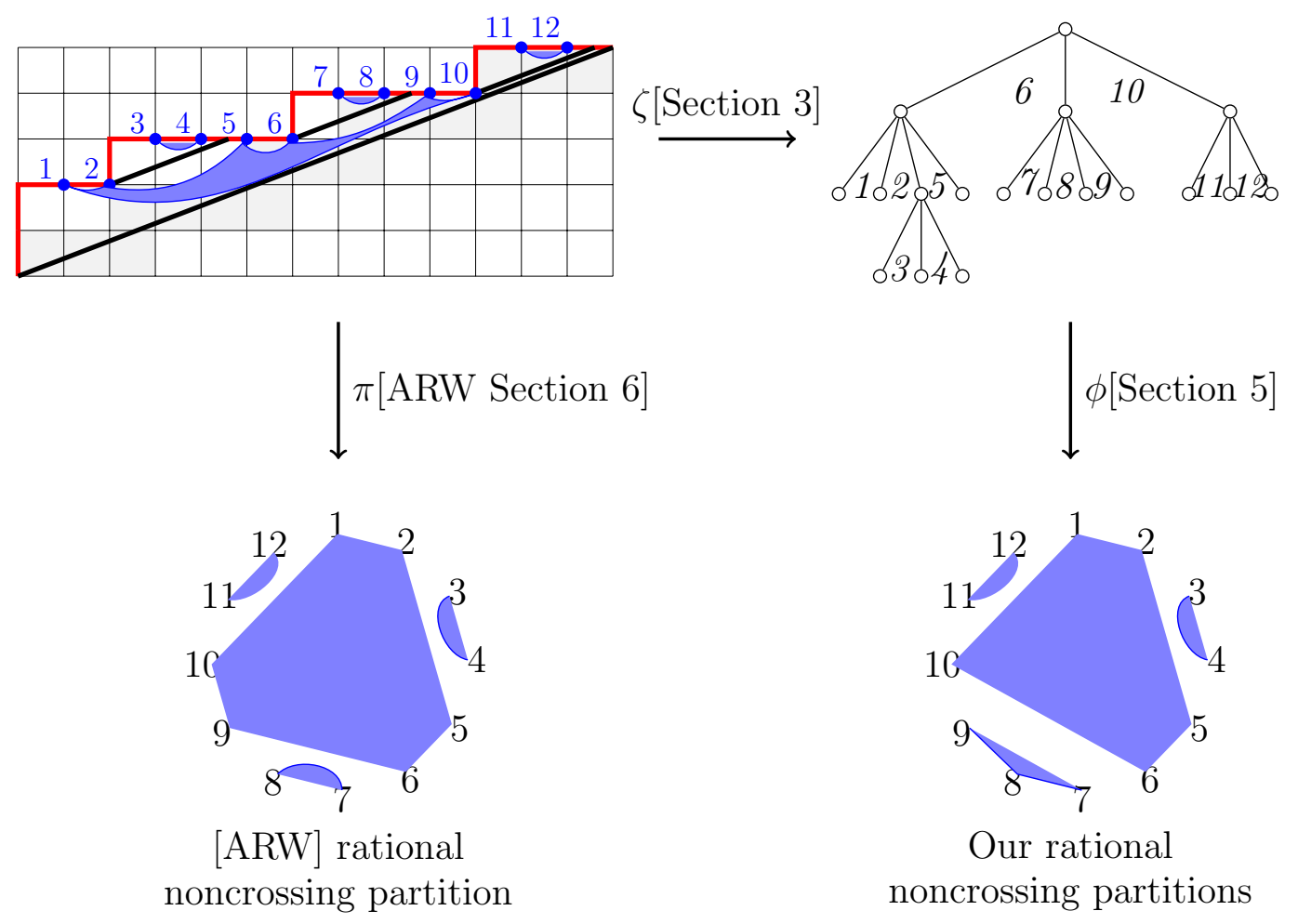

FiguRE 29. Difference between $\widetilde{\mathcal{N C}}_{(a, b)}$ and $\mathcal{N C}_{s}$ for a rational $s$.

the reader will find every meaning clear from the context) such that for an $\mathbb{N}$-module $M=\bigoplus_{n \geq 1} M_{n}$ we have that

$$
\mathcal{T}(M)=\bigoplus \bigoplus_{n \geq 1} \bigotimes_{T \in \mathcal{T}_{n}} \bigotimes_{v \in \operatorname{IntVert}(T)} M_{\operatorname{indeg}(v)}
$$

where $\mathcal{T}_{n}$ is the set of planar rooted trees with $n$ leaves, for $T \in \mathcal{T}_{n}$ we have that $\operatorname{Int} V \operatorname{ert}(T)$ is the set of internal vertices of $T$ and for $v \in \operatorname{Int} V \operatorname{ert}(T)$ we have that $\operatorname{deg}(v)$ is the number of children of $v$. The rule of composition $\gamma: \mathcal{T} \circ \mathcal{T} \rightarrow \mathcal{T}$ is given by composition of trees, i.e., if $T \in \mathcal{T}_{k}$ and $T_{i} \in \mathcal{T}_{m_{i}}$ for $i \in[k]$ then

$$
\gamma\left(T ; T_{1}, T_{2}, \ldots, T_{k}\right)
$$

is the tree in $\mathcal{T}_{\sum_{i} m_{i}}$ constructed by attaching the root of $T_{i}$ to the $i$-th leaf of $T$ from left to right. The identity $\eta: \mathbb{I} \rightarrow \mathcal{T}$ is the natural transformation that maps $\mathbb{I}(M)=M$ to $\eta(\mathbb{I}(M))=M$ that can be seen as having the same form of equation but for each value of $n$ the only tree in $\mathcal{T}_{n}$ to be considered is the corolla with arity $n$, that is the, unique planar rooted tree with signature $(n)$. Algebras over the monad $\mathcal{T}$ are known as nonsymmetric operads and for any $M \in \mathbb{N}$-Mod we have that $\mathcal{T}(M)$ is the free nonsymmetric operad 
generated by $M$. In particular when $M=\bigoplus_{n \geq 1} \mathbf{k}$ then we obtain a free nonsymmetric operad structure on planar rooted trees, see [21].

Note that if $\operatorname{signat}(T)=s$ and $\operatorname{signat}\left(T_{i}\right)=s_{i}$ for $i \in[k]$ then it is not possible to determine exactly $\operatorname{signat}\left(\gamma\left(T ; T_{1}, T_{2}, \ldots, T_{k}\right)\right.$ since it will depend on the tree structure of $T$. However, since as multisets the components of the signatures are preserved we can define a multiset grading on $\mathcal{T}$. Let $[s]$ indicate the underlying multiset associated to a composition $s$. Then for a multiset $S$ we have define

$$
T_{S}=\{T \in \mathcal{T} \mid[\operatorname{signat}(T)] \in S\}
$$

We then have that

$$
\mathcal{T}=\bigcup_{S \text { a multisubset of } \mathbb{N}} T_{S}
$$

with the property that if $T \in T_{S}$ and $T_{i} \in T_{S_{i}}$ for $i \in[k]$ then

$$
\gamma\left(T ; T_{1}, T_{2}, \ldots, T_{k}\right) \in T_{S \cup \cup_{i} S_{i}} .
$$

There is another operadic structure that can be associated to the set $\mathcal{T}$ where composition has a more predictable behavior on signatures. Following a similar idea than in the work of Lopez, Preville-Ratelle and Ronco in [22] on composition on what they call $m$-Dyck paths, we can define a composition of $s$-Dyck paths or their corresponding $s$-trees. In [22] an $m$-Dyck path is defined as a lattice path from $(0,0)$ to $(n m, n m)$, taking only north steps $(m, 0)$ and east steps $(0,1)$ and such that $m$ times the number of north steps taken at any given point is larger than the number of east steps taken. Let us denote the set of $m$-Dyck paths by $\mathcal{D} y c k^{m}$. There is an almost trivial bijection between $\mathcal{D} y c k^{m}$ and $\mathcal{D P}{ }_{\left((m+1)^{n}\right)}$ by just keeping the same word in the letters $N$ and $E$ that describes $D \in \mathcal{D} y c k^{m}$ and adding a trailing $E$ to get a path in $\mathcal{D} \mathcal{P}_{\left((m+1)^{n}\right)}$, i.e., if $D=N E^{i_{1}} N E^{i_{2}} \cdots N E^{i_{a}} \in$ $\mathcal{D}_{y c k^{m}}$ if and only if $D E=N E^{i_{1}} N E^{i_{2}} \cdots N E^{i_{a}} E \in \mathcal{D} \mathcal{P}\left((m+1)^{n}\right)$. Composition is defined in [22] as follows: let $D, D_{0}, D_{1} \cdots, D_{k} \in \mathcal{D} y c k^{m}$ such that the NE word associated to $D=N E^{i_{1}} N E^{i_{2}} \cdots N E^{i_{a}}$ has $i_{a}=k$, then the composition is defined as

$$
\tilde{\gamma}\left(D ; D_{0}, D_{1}, \cdots, D_{k}\right)=N E^{i_{1}} N E^{i_{2}} \cdots N D_{0} E D_{1} E \cdots E D_{k} .
$$

From the perspective of $s$-trees under the bijection of Theorem 3.15, it can be shown that this composition can be seen as a special case of the composition

$$
\gamma\left(T ; \bullet, \bullet, \cdots, \bullet, T_{0}, T_{2}, \ldots, T_{k}\right),
$$

where the only non-identity trees substituted in $T$ are attached to leaves of $T$ that follow the last internal node when the nodes are ordered in preorder. Under this conditions it then follows that

$$
\operatorname{signat}\left(\gamma\left(T ; \bullet, \bullet, \cdots, \bullet, T_{0}, T_{2}, \ldots, T_{k}\right)\right)=\operatorname{signat}(T) \oplus \operatorname{signat}\left(T_{0}\right) \oplus \cdots \oplus \operatorname{signat}\left(T_{k}\right),
$$

and hence the composition in this operadic structure behaves well under concatenation of signatures. Here $\mathcal{T}=\bigcup_{s \in \mathcal{C} \text { omp }} \mathcal{T}_{s}$ does not have an arity grading anymore but instead a grading in terms of the length $\ell(s)$ of the signature. 
Finally, algebraic structures can be associated to the two operadic structures discussed above to give constructions related to the Hopf algebras of Loday and Ronco for planar binary trees [20], the Hopf algebras of Novelli and Thibon for $(m+1)$-ary trees [24] and the operadic constructions on $\mathcal{D} y c k^{m}[22]$.

\section{Appendix A. Bijections with $s$-Dyck PAThs}

In this appendix we describe further bijections between $s$-Catalan objects and $s$-Dyck paths. As in the case of $s$-trees, these bijections turn out to be elegant and simple (see Figure 30). They are obtained by assigning some labels to an $s$-Dyck path, and then reading or grouping the labels according to a certain rule. The bijections presented here are in accordance to those presented in Section 3. More precisely, if $D$ is the $s$-Dyck path corresponding to an $s$-tree $T$ (according to Section 3.2.1), then an $s$-Catalan object associated to $D$ in this appendix is the same $s$-Catalan object associated to $T$ according to the corresponding bijection in Section 3. All the details about these bijections are simple and left to be filled by the interested reader.

A.1. $s$-Dyck paths and 312-avoiding Stirling $(s-1)$-permutations. Let $\sigma$ be an $(s-\mathbf{1})$-permutation. We represent the permutation in a $(|s|-\ell(s)+1) \times \ell(s)$ rectangle by placing a dot in the square at position $(i, j)$ if $\sigma(i)=j$. Note that all the columns of the rectangle except the last one are occupied. The top part of Figure 30 illustrates an example for the $(2,3,3,1,4)$-permutation $\sigma=2233321155554$. Given such representation of $\sigma$ we shade all the boxes in the rectangle that are weakly below and weakly to the right of some dotted box. It is not hard to see that the shaded boxes bound an $s$-Dyck path $D(\sigma)$. Note that many permutations may give rise the same Dyck path. However, if we restrict to 312-avoiding Stirling $(s-\mathbf{1})$-permutations there is exactly one permutation for each Dyck path. This permutation is constructed as follows: place $s(j)-1$ dots, from left to right, in the highest unoccupied row $j$ that is below the path $D$, such that no previously occupied columns are taken. The permutation $\sigma(D)$ is defined as the permutation with the resulting rectangular representation.

A.2. $s$-Dyck paths and noncrossing $(s-1)$-partitions. Given an $s$-Dyck path $D$ we place $s(j)-1$ dots in row $j$ for each $j$ as above, and number the columns of the grid (except the last one) from 1 to $|s|-\ell(s)$. We make an horizontal strip to the right of each maximal consecutive chain of north steps in $D$. The columns of the dots in each strip form the blocks of the noncrossing $(s-\mathbf{1})$-partition $\pi(D)$ associated to $D$. The middle part of Figure 30 illustrates an example. Note that the number of blocks of the partition is equal, by definition, to the number of peaks of the Dyck path. The inverse map can be easily described as follows: let $\pi=\left\{\pi_{1}, \ldots, \pi_{k}\right\}$ be a noncrossing $(s-\mathbf{1})$-partition whose blocks are ordered according to the order of their minimal elements. Partition the sequence $s-\mathbf{1}$ in $k$ consecutive blocks such that the sum of the values in block $i$ is equal to the size $\left|\pi_{i}\right|$. In the middle of Figure 30, $s-\mathbf{1}=(\mathbf{2}, \mathbf{3}, \mathbf{3}, \mathbf{1}, \mathbf{4})$ and

$$
\pi=\{\{1,2,6,7,8\},\{3,4,5\},\{9,10,11,12,13\}\} .
$$




\section{Bijections with $s$-Dyck paths}

For all bijections place first $s(i)-1$ dots in row $i$ below the Dyck path from top to bottom and left justified

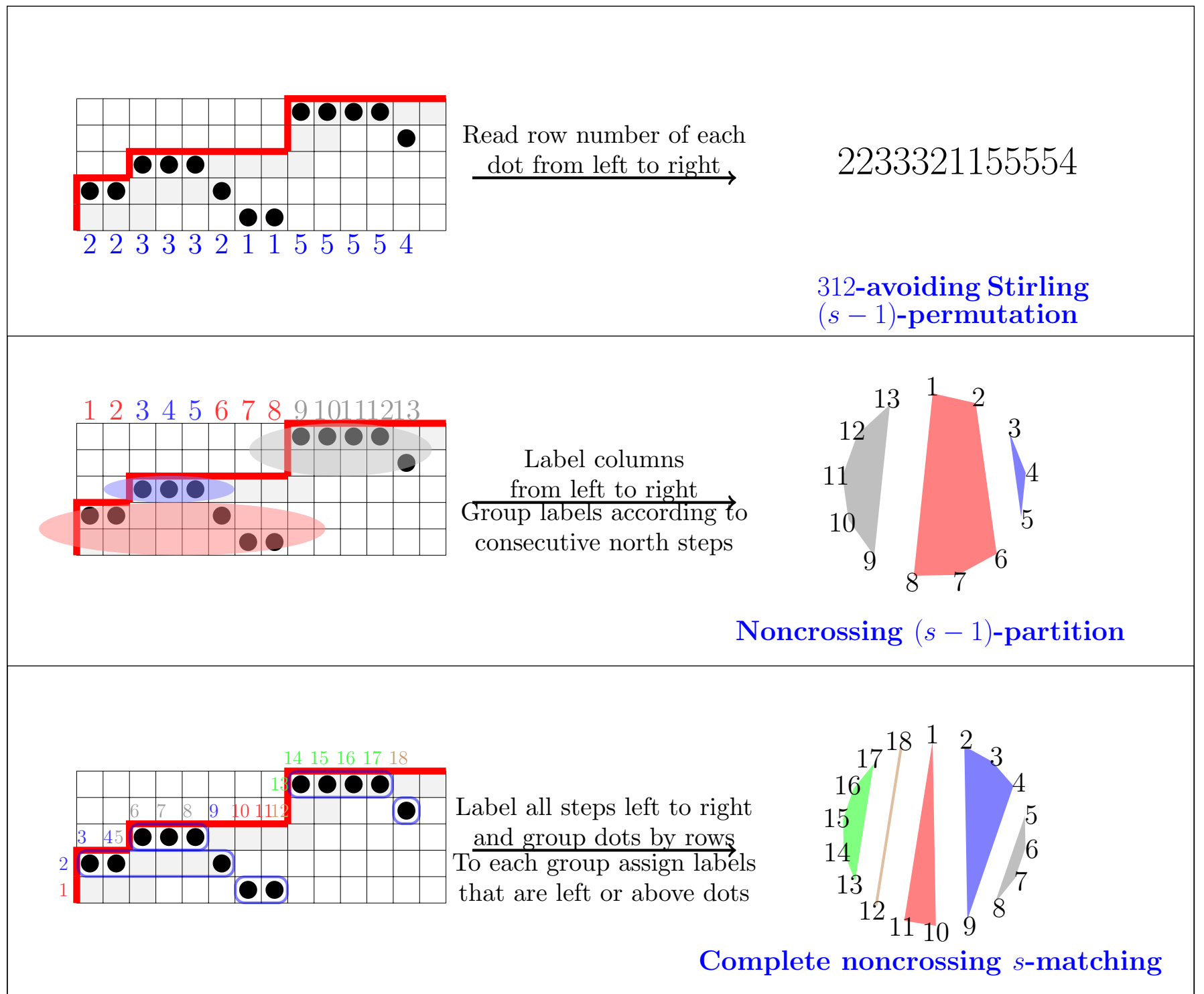

FiguRE 30. Examples of the bijections with $s$-Dyck paths.

The sizes of the blocks of $\pi$ are $(5,3,5)$, and so we partition $s-\mathbf{1}$ as $|2,3| 3|1,4|$. For $1 \leq i \leq k$, define $x_{i}=\min \pi_{i}-1$ and $y_{i}$ equal to the position of the last number in block $i$ 
of the partitioned $s-\mathbf{1}$. In our running example,

$$
\begin{gathered}
\left(x_{1}, x_{2}, x_{3}\right)=(1-1,3-1,9-1)=(0,2,8), \\
\left(y_{1}, y_{2}, y_{3}\right)=(2,3,5) .
\end{gathered}
$$

The $s$-Dyck path corresponding to $\pi$ is the path with peaks at coordinates $\left(x_{i}, y_{i}\right)$ for $i \in[k]$.

A.3. $s$-Dyck paths and complete noncrossing $s$-matchings. Given an $s$-Dyck path $D$, we place $s(j)-1$ dots in row $j$ for each $j$ as above, and number the steps of $D$ (except the last one) from 1 to $|s|$. For each $1 \leq i \leq \ell(s)$, define $M_{i}$ to be the set containing the label of the north step of $D$ in row $i$, together with the labels of the east steps in the columns of the dots in that row. The complete noncrossing $s$-matching associated to $D$ is defined by $M(D):=\left\{M_{1}, \ldots, M_{\ell(s)}\right\}$. The bottom part of Figure 30 illustrates an example. The inverse is determined by the positions of the north steps in the path, which are the minimal elements of the blocks in $M$.

\section{ACKNOWLEDGEMENTS}

This project started in November 2014, during a visit of the second author to the first author at York University and the Fields Institute. We thank the Banting Fellowships Program of the Government of Canada and York University for their financial support and for making this visit possible. We also thank Robin Sulzgruber for his input in the description of the bijection between $s$-Dyck paths and complete noncrossing $s$-matchings in Section A.3; and Nantel Bergeron for the many useful conversations.

\section{REFERENCES}

[1] Drew Armstrong. Generalized noncrossing partitions and combinatorics of Coxeter groups. Mem. Amer. Math. Soc., 202(949):x+159, 2009.

[2] Drew Armstrong, Nicholas A. Loehr, and Gregory S. Warrington. Rational parking functions and Catalan numbers. Ann. Comb., 20(1):21-58, 2016.

[3] Drew Armstrong, Brendon Rhoades, and Nathan Williams. Rational associahedra and noncrossing partitions. Electron. J. Combin., 20(3):Paper 54, 27, 2013.

[4] Christos A. Athanasiadis. On a refinement of the generalized Catalan numbers for Weyl groups. Trans. Amer. Math. Soc., 357(1):179-196, 2005.

[5] Cesar Ceballos and Viviane Pons. The s-weak order and the s-permutahedron. work in progress, 2018.

[6] Rafael S González D'León. A family of symmetric functions associated with Stirling permutations. arXiv preprint arXiv:1506.01628, 2015.

[7] Sergey Fomin and Nathan Reading. Generalized cluster complexes and Coxeter combinatorics. Int. Math. Res. Not., (44):2709-2757, 2005.

[8] A. M. Garsia and M. Haiman. A remarkable $q$,t-Catalan sequence and $q$-Lagrange inversion. J. Algebraic Combin., 5(3):191-244, 1996.

[9] Petar Gaydarov and Sam Hopkins. Parking functions and tree inversions revisited. Adv. in Appl. Math., 80:151-179, 2016.

[10] Ira Gessel and Richard P. Stanley. Stirling polynomials. J. Combinatorial Theory Ser. A, 24(1):24-33, 1978.

[11] Ronald L Graham, Donald Ervin Knuth, and Oren Patashnik. Concrete mathematics: a foundation for computer science, volume 2. Addison-Wesley Reading, MA, 1994. 
[12] António Guedes de Oliveira and Michel Las Vergnas. Parking functions and labeled trees. Sém. Lothar. Combin., 65:Art. B65e, 10, 2010/12.

[13] Svante Janson, Markus Kuba, and Alois Panholzer. Generalized Stirling permutations, families of increasing trees and urn models. J. Combin. Theory Ser. A, 118(1):94-114, 2011.

[14] Alan G Konheim and Benjamin Weiss. An occupancy discipline and applications. SIAM Journal on Applied Mathematics, 14(6):1266-1274, 1966.

[15] G. Kreweras. Une famille de polynômes ayant plusieurs propriétés énumeratives. Period. Math. Hungar., 11(4):309-320, 1980.

[16] Germain Kreweras. Sur une classe de problemes de dénombrement liés au treillis des partitions des entiers. Cahiers du Bureau universitaire de recherche opérationnelle Série Recherche, 6:9-107, 1965.

[17] Markus Kuba and Alois Panholzer. Analysis of statistics for generalized Stirling permutations. Combin. Probab. Comput., 20(6):875-910, 2011.

[18] Romuald Lenczewski. Limit distributions of random matrices. Adv. Math., 263:253-320, 2014.

[19] Romuald Lenczewski and RafatSał apata. Multivariate Fuss-Narayana polynomials and their application to random matrices. Electron. J. Combin., 20(2):Paper 41, 14, 2013.

[20] Jean-Louis Loday and María O. Ronco. Hopf algebra of the planar binary trees. Adv. Math., 139(2):293-309, 1998.

[21] Jean-Louis Loday and Bruno Vallette. Algebraic operads, volume 346 of Grundlehren der Mathematischen Wissenschaften [Fundamental Principles of Mathematical Sciences]. Springer, Heidelberg, 2012.

[22] Daniel López, Louis-François Préville-Ratelle, and María Ronco. Algebraic structures defined on $m$ Dyck paths. arXiv preprint arXiv:1508.01252, 2015.

[23] Clauda Malvenuto and Christophe Reutenauer. Duality between quasi-symmetric functions and the Solomon descent algebra. J. Algebra, 177(3):967-982, 1995.

[24] J.-C. Novelli and J.-Y. Thibon. Hopf Algebras of m-permutations, $(\mathrm{m}+1)$-ary trees, and m-parking functions. arXiv:1403.5962 [math], March 2014. arXiv: 1403.5962.

[25] SeungKyung Park. Inverse descents of $r$-multipermutations. Discrete Math., 132(1-3):215-229, 1994.

[26] SeungKyung Park. P-partitions and $q$-Stirling numbers. J. Combin. Theory Ser. A, 68(1):33-52, 1994.

[27] SeungKyung Park. The r-multipermutations. J. Combin. Theory Ser. A, 67(1):44-71, 1994.

[28] David Perkinson, Qiaoyu Yang, and Kuai Yu. G-parking functions and tree inversions. Combinatorica, pages 1-14, 2016.

[29] T Kyle Petersen. Eulerian numbers. In Eulerian Numbers, pages 3-18. Springer, 2015.

[30] Viviane Pons. A lattice on decreasing trees : the metasylvester lattice. In 27th International Conference on Formal Power Series and Algebraic Combinatorics (FPSAC 2015), Discrete Math. Theor. Comput. Sci. Proc., AS, pages 381-392. Assoc. Discrete Math. Theor. Comput. Sci., Nancy, 2015.

[31] Victor Reiner. Non-crossing partitions for classical reflection groups. Discrete Math., 177(1-3):195-222, 1997.

[32] Jeffrey B. Remmel and Andrew Timothy Wilson. Block patterns in Stirling permutations. J. Comb., 6(1-2):179-204, 2015.

[33] Richard P. Stanley. Parking functions and noncrossing partitions. Electron. J. Combin., 4(2):Research Paper 20, approx. 14 pp. (electronic), 1997. The Wilf Festschrift (Philadelphia, PA, 1996).

[34] Richard P. Stanley. Enumerative combinatorics. Volume 1, volume 49 of Cambridge Studies in Advanced Mathematics. Cambridge University Press, Cambridge, second edition, 2012.

[35] Richard P. Stanley. Catalan Numbers. Cambridge University Press, March 2015.

[36] Richard P. Stanley and Jim Pitman. A polytope related to empirical distributions, plane trees, parking functions, and the associahedron. Discrete Comput. Geom., 27(4):603-634, 2002.

[37] Nathan Williams. Cataland. ProQuest LLC, Ann Arbor, MI, 2013. Thesis (Ph.D.)-University of Minnesota. 
[38] Catherine H. Yan. Generalized parking functions, tree inversions, and multicolored graphs. Adv. in Appl. Math., 27(2-3):641-670, 2001. Special issue in honor of Dominique Foata's 65th birthday (Philadelphia, PA, 2000).

[39] Catherine Huafei Yan. On the enumeration of generalized parking functions. In Proceedings of the Thirty-first Southeastern International Conference on Combinatorics, Graph Theory and Computing (Boca Raton, FL, 2000), volume 147, pages 201-209, 2000.

Faculty of Mathematics, University of Vienna, Vienna, Austria

E-mail address: cesar.ceballos@univie.ac.at

Escuela de Ciencias Exactas e Ingeniería, Universidad Sergio Arboleda, Bogotá, ColomBIA

E-mail address: rafael.gonzalezl@usa.edu.co 